\title{
Cardioprotective Potentials of Plant-Derived Small Molecules against Doxorubicin Associated Cardiotoxicity
}

\author{
Shreesh Ojha, ${ }^{1}$ Hasan Al Taee, ${ }^{1}$ Sameer Goyal, ${ }^{2}$ Umesh B. Mahajan, ${ }^{2}$ \\ Chandrgouda R. Patil, ${ }^{2}$ D. S. Arya, ${ }^{3}$ and Mohanraj Rajesh ${ }^{1}$ \\ ${ }^{1}$ Department of Pharmacology and Therapeutics, College of Medicine and Health Sciences, United Arab Emirates University, \\ P.O. Box 17666, Al Ain, UAE \\ ${ }^{2}$ Department of Pharmacology, R. C. Patel Institute of Pharmaceutical Education and Research, Shirpur, Maharashtra 425405, India \\ ${ }^{3}$ Department of Pharmacology, All India Institute of Medical Sciences, New Delhi 110029, India
}

Correspondence should be addressed to Mohanraj Rajesh; rajm@uaeu.ac.ae

Received 8 January 2016; Revised 2 April 2016; Accepted 20 April 2016

Academic Editor: Sidhartha D. Ray

Copyright (c) 2016 Shreesh Ojha et al. This is an open access article distributed under the Creative Commons Attribution License, which permits unrestricted use, distribution, and reproduction in any medium, provided the original work is properly cited.

\begin{abstract}
Doxorubicin (DOX) is a potent and widely used anthracycline antibiotic for the treatment of several malignancies. Unfortunately, the clinical utility of DOX is often restricted due to the elicitation of organ toxicity. Particularly, the increased risk for the development of dilated cardiomyopathy by DOX among the cancer survivors warrants major attention from the physicians as well as researchers to develop adjuvant agents to neutralize the noxious effects of DOX on the healthy myocardium. Despite these pitfalls, the use of traditional cytotoxic drugs continues to be the mainstay treatment for several types of cancer. Recently, phytochemicals have gained attention for their anticancer, chemopreventive, and cardioprotective activities. The ideal cardioprotective agents should not compromise the clinical efficacy of DOX and should be devoid of cumulative or irreversible toxicity on the naïve tissues. Furthermore, adjuvants possessing synergistic anticancer activity and quelling of chemoresistance would significantly enhance the clinical utility in combating DOX-induced cardiotoxicity. The present review renders an overview of cardioprotective effects of plant-derived small molecules and their purported mechanisms against DOX-induced cardiotoxicity. Phytochemicals serve as the reservoirs of pharmacophore which can be utilized as templates for developing safe and potential novel cardioprotective agents in combating DOX-induced cardiotoxicity.
\end{abstract}

\section{Introduction}

Doxorubicin (DOX) is a potent and widely used anthracycline antibiotic for the treatment of cancers. However, the major impeding issue pertaining to the clinical application of DOX is related to its ability to induce untoward toxicity to the healthy tissues [1]. The occurrence of fatal cardiotoxicity in pediatric as well as in adult patients is characterized by an irreversible cardiomyopathy which compromises the clinical utility of DOX and accounts for the major cause of the chemotherapy related morbidity and mortality [1]. In spite of introducing several less toxic derivatives of DOX, elicitation of cardiotoxicity still remains the major concern [2]. However, with the advent of newer class of monoclonal antibodies revolutionized cancer chemotherapy, still this approach is burdened with myriad adverse effects [3]. Thus, the use of traditional cytotoxic drugs continues to be a preferred mode for the treatment of cancer.

To limit the DOX-induced cardiotoxicity, several molecules, such as beta blockers, angiotensin receptor blockers, amifostine, dexrazoxane, Mesna (2-mercaptoethane sulfonate $\mathrm{Na}$ ), leucovorin, and erythropoietin, have been evaluated as cardioprotective adjuvants in preclinical studies [4]. Recently, dexrazoxane, when subjected to clinical trial against combating DOX-induced cardiotoxicity, exhibited marked cardioprotection and did not compromise the anticancer activity of DOX [5]. Similarly, carvedilol (beta blocker) has also been demonstrated to confer protection against DOX-induced cardiotoxicity in human subjects [6]. However, large-scale clinical applications of these adjuvants 


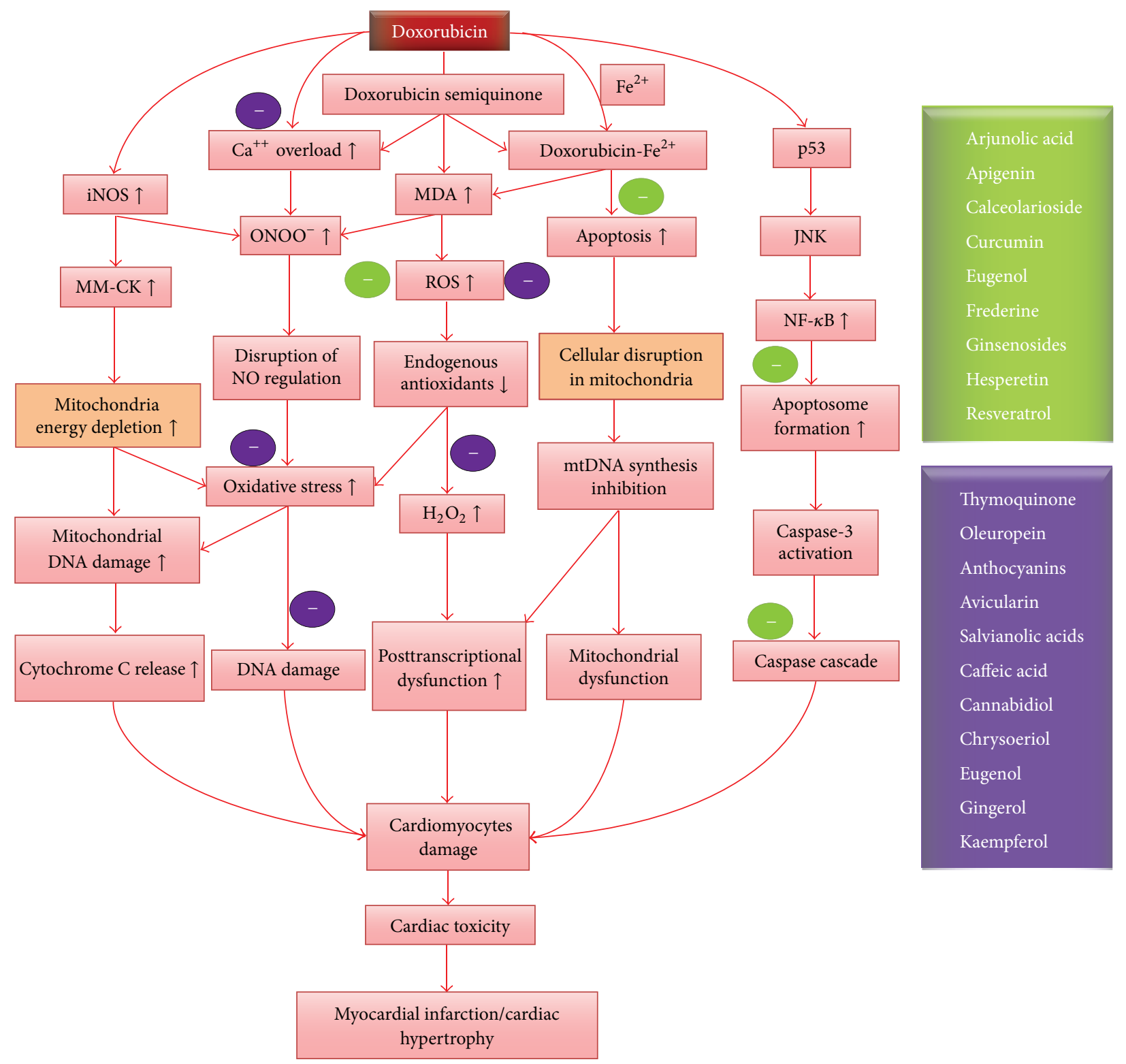

FIGURE 1: This scheme shows the pathways involved in the elicitation of DOX-induced adverse effects in the myocardium and its attenuation by phytochemicals.

are yet to be established in human subjects. The substantial burden arising from cancer and cardiotoxicity and their interrelationship in imposing morbidity and mortality has emerged as the major driving factor for the academia and pharmaceutical industry to devise and develop strategies that can simultaneously provide long-term cardioprotection from DOX-associated cardiotoxicity without compromising the efficacy of cancer chemotherapy [7]. Since the origin of the human civilization, plants and herbs have been traditionally used in the treatment of various diseases and ailments [8]. In this direction, the prospect of harnessing the potentials of plant-derived small molecules (phytochemicals) appears to provide rich dividends, since phytochemicals have been extensively studied in the preclinical studies and shown to possess anti-inflammatory, antioxidant, and anticancer activities. Phytochemicals are the natural constituents of herbs and plants. Moreover, anticancer drug paclitaxel and antimalarial agent artemisinin are phytochemicals originally extracted from plants; however, these agents are now chemically synthesized $[9,10]$.

In this review, we have systematically presented the evidence wherein the phytochemicals were investigated for cardioprotective effects against DOX and the relevant mechanisms. Several mechanisms have been postulated for the development of DOX-induced cardiotoxicity. However, oxidative stress driven inflammation, apoptosis, and myocardial remodeling have emerged to be the key players in DOXinduced cardiotoxicity. In-depth description of pathophysiology of DOX-induced cardiotoxicity has been reviewed elsewhere [11, 12]; however, to provide clarity to our discussion, we have provided a simplified scheme for the pathomechanisms in Figure 1. 


\section{Phytochemicals Limiting DOX-Induced Cardiotoxicity}

In this section, we systematically described the phytochemicals investigated for their cardioprotective activity against DOX-induced toxic effects in the myocardium. Phytochemicals investigated in the animal model of DOX-induced cardiotoxicity are listed in Table 1 and the molecules investigated in cell culture models (in vitro) are presented in Table 2.

2.1. Arjunolic Acid. Arjunolic acid is a chiral triterpenoid saponin, isolated from Terminalia arjuna. Arjunolic acid treatment to adult rat cardiomyocytes in the presence of DOX attenuated caspase-dependent apoptotic signaling by ameliorating proapoptotic $\mathrm{p} 53, \mathrm{p} 38$, and JNK-MAPKs and mitochondrial pathways leading to apoptosis. Furthermore, arjunolic acid when administered to rats significantly inhibited DOX-induced myocardial toxicity by mitigating oxidative stress and apoptotic pathways [13].

2.2. Anthocyanins. Anthocyanins are a group of polyphenolic compounds which are abundantly found in common fruits and vegetables. The effect of six anthocyanidins (cyanidin chloride, delphinidin chloride, malvidin chloride, pelargonidin chloride, peonidin chloride, and petunidin chloride) and seven anthocyanins (cyanidin $3-\mathrm{O}-\beta$-galactopyranoside chloride, cyanidin 3-O- $\beta$-glucopyranoside chloride, delphinidin 3-O- $\beta$-glucopyranoside chloride, malvidin 3-O- $\beta$-glucopyranoside chloride, pelargonidin 3 -O- $\beta$-glucopyranoside chloride, peonidin 3 -O- $\beta$-glucopyranoside chloride, and petunidin 3-O- $\beta$-glucopyranoside chloride) was investigated against DOX-induced cardiotoxicity in $\mathrm{H} 9 \mathrm{c} 2$ cardiomyoblasts. All these anthocyanidins improved the cell viability via quenching of reactive oxygen species (ROS) [14]. Delphinidin was found to confer protection against DOX and etoposide inhibition of topoisomerase II, thus warranting careful scrutiny against the use of these agents in combating DOX-induced cardiotoxicity.

2.3. Apigenin. Apigenin is a flavone type flavonoid predominantly found in flowers of chamomile. It is also present in edible plants such as fruits (oranges, apples, cherries, and grape fruits) and vegetables (onions, celery, parsley, broccoli, pepper, barley, and tomatoes) [15]. Apigenin treatment to adult rat cardiomyocytes significantly improved cell viability in the presence of DOX and the mechanism appeared to involve quenching of ROS, mitigation of lipid peroxidation, and myocyte necrosis [16]. However, to date, no studies have demonstrated apigenin efficacy in vivo.

2.4. Avicularin. Avicularin, chemically a biflavonoid and a quercetin glycoside, is isolated from the leaves of a flowering plant, Malus hupehensis, popularly known as Chinese crabapple, Hupeh crab, or tea crabapple [17]. The protective effects of avicularin against DOX-induced cardiotoxicity were demonstrated in $\mathrm{H} 9 \mathrm{c} 2$ cells and the mechanism purported was via its antioxidant property [17]. However, the cardioprotective effects of avicularin are yet to be confirmed in vivo.

2.5. Berberine. Berberine is an alkaloid identified in the roots and bark of the Berberis species [18]. For the first time, its protective effects on DOX-induced cardiotoxicity in mice were studied by Lv et al. [19]. Berberine was found to reduce mortality, improve body weight and cardiac function, and restore ECG changes in DOX-treated rats. Furthermore, in neonatal rat cardiomyocytes, berberine inhibited DOXinduced apoptosis via counteracting ROS induced p53 activation, mitochondrial dissipation, executioner caspase activation, and activation of AMPK [19]. Moreover, berberine has also been shown to inhibit DOX-induced cardiotoxicity via mitigation of biotransformation of DOX, thereby limiting the bioavailability of doxorubicinol (a major alcohol metabolite of DOX) in the cytoplasm of rat hearts [20].

2.6. Baicalein. Baicalein is a flavonoid derived from the roots of Scutellaria baicalensis [21]. By using cultured chick cardiomyocytes in vitro, it was shown that baicalein attenuated DOX-induced ROS activation, proapoptotic MAPK, and apoptosis [22]. Recently, in a murine model of DOXinduced cardiotoxicity, baicalein significantly mitigated cardiac injury via augmenting nuclear factor E2-related factor2 (Nrf2), antioxidant defense, blunting of nitrative stress, inflammation, and apoptosis [23].

2.7. Caffeic Acid Phenethyl Ester (CAPE). Caffeic acid phenethyl ester is an active component of propolis which is the major hive product of bees and is known to be rich in flavonoids [24]. CAPE has been demonstrated to attenuate DOX-induced cardiotoxicity via attenuation of ROS generation and apoptosis. Furthermore, it also improved cardiac function as assessed by hemodynamic measurements and preserved the myocardial structure [25].

2.8. Calceolarioside. Calceolarioside is a phenylpropanoid glycoside isolated from Calceolaria hypericina known to possess antiplatelet and anticancer activities [26]. Calceolarioside attenuated DOX-induced cardiotoxicity in H9c2 cells via upregulation of antioxidant enzymes and suppression of apoptosis [27]. However, these effects are yet to be confirmed in animal models.

2.9. Cannabidiol. Cannabidiol (CBD) is a major nonpsychoactive constituent of the plant Cannabis sativa, popularly known as Marijuana and used for recreational as well as medicinal purposes [28]. In a chronic model of DOXinduced cardiotoxicity in rats, $\mathrm{CBD}$ has been demonstrated to suppress myocardial toxicity via attenuating oxidative stress, inflammation, and cell death pathways [29]. Recently, Hao et al. demonstrated that CBD attenuated DOX-induced cardiotoxicity via augmenting mitochondrial biogenesis and blunting of oxidative and nitrative stress and apoptosis [30]. It is also pertinent to note that CBD also exerts several cardioprotective actions against diabetic cardiovascular complications [31] and it has been approved in Canada and 
TABLE 1: Phytochemicals investigated for cardioprotective activity against DOX-induced cardiotoxicity in in vivo studies.

\begin{tabular}{|c|c|c|c|}
\hline Phytochemical & $\begin{array}{l}\text { DOX-induced } \\
\text { cardiomyopathy- } \\
\text { animal model } \\
\text { (acute or chronic) }\end{array}$ & $\begin{array}{l}\text { Cardiac function } \\
\text { determined } \\
(\text { yes/no })\end{array}$ & References \\
\hline Arjunolic acid & Chronic & Yes & {$[13]$} \\
\hline Berberine & Chronic & Yes & [20] \\
\hline Berberine & Acute & Yes & {$[19]$} \\
\hline Baicalein & Chronic & No & [23] \\
\hline Caffeic acid phenethyl ester & Acute & Yes & [25] \\
\hline Cannabidiol & Acute & Yes & [30] \\
\hline Cannabidiol & Chronic & No & {$[29]$} \\
\hline Carotenoids & Acute & No & {$[34]$} \\
\hline Eugenol & Acute & Yes & {$[51]$} \\
\hline Gingerol & Chronic & No & {$[56]$} \\
\hline 23-Hydroxybetulinic acid & Chronic & Yes & {$[60]$} \\
\hline Hesperetin & Chronic & No & {$[64]$} \\
\hline Hesperidin & Acute & No & {$[62]$} \\
\hline Isorhamnetin & Chronic & No & {$[66]$} \\
\hline Indole-3-carbinol & Chronic & No & [67] \\
\hline Kaempferol & Chronic & No & {$[69]$} \\
\hline Lycopene & Acute & No & {$[70]$} \\
\hline Lycopene & Acute & No & {$[71]$} \\
\hline Lycopene & Acute & Yes & {$[72]$} \\
\hline Mangiferin & Acute & No & [79] \\
\hline Mangiferin & Acute & No & {$[78]$} \\
\hline Naringenin & Acute & No & [79] \\
\hline Naringenin & Acute & No & {$[80]$} \\
\hline Ocotillol & Acute & No & {$[86]$} \\
\hline Ocotillol & Chronic & No & {$[86]$} \\
\hline Hydroxytyrosol & Chronic & No & {$[65]$} \\
\hline Tetrandrine & Chronic & Yes & {$[140]$} \\
\hline Periplogenin & Chronic & No & {$[92]$} \\
\hline p-coumaric acid & Acute & No & {$[82]$} \\
\hline Procyanidins & Chronic & Yes & {$[100]$} \\
\hline Robinin & Acute & No & {$[114]$} \\
\hline Thymoquinone & Acute & No & {$[137]$} \\
\hline Thymoquinone & Acute & No & [138] \\
\hline Silibinin & Chronic & Yes & {$[126]$} \\
\hline Sesamin & Acute & No & {$[124]$} \\
\hline Sesamol & Chronic & No & {$[125]$} \\
\hline $\begin{array}{l}\text { Tetrahydroxystilbene } \\
\text { glucoside }\end{array}$ & Acute & No & [135] \\
\hline Oleuropein & Acute & No & [87] \\
\hline Oleuropein & Acute & No & {$[88]$} \\
\hline Oleuropein & Chronic & Yes & [89] \\
\hline Frederine & Acute & Yes & {$[52]$} \\
\hline
\end{tabular}


TABle 1: Continued.

\begin{tabular}{|c|c|c|c|}
\hline Phytochemical & $\begin{array}{l}\text { DOX-induced } \\
\text { cardiomyopathy- } \\
\text { animal model } \\
\text { (acute or chronic) }\end{array}$ & $\begin{array}{l}\text { Cardiac function } \\
\text { determined } \\
(\text { yes } / \text { no })\end{array}$ & References \\
\hline Visnagin & Acute & Yes & [146] \\
\hline Visnagin & Chronic & Yes & [146] \\
\hline Schisandrin B & Acute & Yes & {$[121]$} \\
\hline Schisandrin B & Chronic & Yes & [122] \\
\hline Salvianolic acid A & Acute & Yes & [117] \\
\hline Tanshinone IIA & Chronic & Yes & [133] \\
\hline Oleuropein & Acute & No & [87] \\
\hline Oleuropein & Acute & No & {$[88]$} \\
\hline Oleuropein & Chronic & Yes & [89] \\
\hline Frederine & Acute & Yes & {$[52]$} \\
\hline Visnagin & Acute & Yes & [146] \\
\hline Visnagin & Chronic & Yes & [146] \\
\hline Schisandrin B & Acute & Yes & [121] \\
\hline Schisandrin B & Chronic & Yes & {$[122]$} \\
\hline Salvianolic acid A & Acute & Yes & {$[117]$} \\
\hline Tanshinone IIA & Chronic & Yes & {$[133]$} \\
\hline
\end{tabular}

Europe for the management of pain associated with multiple sclerosis [32].

2.10. Carotenoids. Carotenoids are the organic pigments and constitute a large group of more than 600 compounds found in plants, which impart color to the leaves and fruits [33]. These are produced from 8 isoprene molecules and contain 40 carbon atoms and are also known as tetraterpenoids with polyisoprenoid structure having a long conjugated double bond system forming the backbone of the molecule, which may be terminated by cyclic end groups that contain oxygenbearing substitutes. The electron-rich conjugated system of the polyene is believed to afford antioxidant and free radical scavenging activity and these pharmacological effects were attributed to health benefits offered by carotenoids [33]. Recently, the cardioprotective efficacy of carotenoids was demonstrated in DOX-induced tumor-bearing mice [34]. Specifically, carotenoids were found to improve the antioxidant defense and preserve the myocardial membranes, reflected as reduced leakage of myocyte injury marker enzymes without compromising DOX activity on the tumor growth inhibition [34].

2.11. Chrysin. Chrysin is a flavone class of flavonoid and one of the most important bioactive constituents of different fruits, vegetables, and mushrooms [35]. Recently, chrysin cardioprotective effect against DOX-induced acute cardiotoxicity in rats was demonstrated by Mantawy et al. [36]. Chrysin was found to improve antioxidant defense, attenuate oxidative/nitrative stress, and suppress the generation of inflammatory mediators [36].
2.12. Catechins. Epigallocatechin-3-gallate (EGCG) accounts for $50-80 \%$ of catechins in green tea and represents about 200-300 mg in a brewed cup of green tea. Convincing data are available to demonstrate that catechins possess potent antioxidant, anti-inflammatory, immunomodulatory, cardioprotective, and anticancer activities [37]. Green tea leaf extract supplementation in cultured rat cardiomyocytes showed its ability to protect the cells against DOX-induced decreased H9c2 cells viability, via quenching of ROS generation [38]. EGCG in vitro has been demonstrated to protect cardiomyocytes of neonatal rat hearts from DOX-induced cytotoxicity by attenuating ROS production, apoptosis, and increasing activities and protein expression of endogenous antioxidant enzymes [39]. In another study, EGCG treatment to rat cardiomyocytes significantly attenuated DOX-induced ROS generation and alterations in myocyte contractile dynamics via modulation of proteins involved in calcium handling system [40]. In addition, EGCG also elicited cardioprotective effects on a chronic model of DOX-induced cardiotoxicity via attenuation of oxidative stress and apoptosis pathways [41].

It has been reported that supplementation of EGCG in the diet increases the activities of P-450 family of reductase, augments the bioavailability of DOX, and could predispose the subject to increased risk of cardiotoxicity [42]. Hence, further studies are warranted to investigate and extend the cardioprotective benefits of ECGG, without untoward perpetuation of DOX-induced cardiotoxicity.

2.13. Chrysoeriol. Chrysoeriol is a flavone compound isolated from the leaves of Digitalis purpurea, popularly known as Foxglove and well reputed for its cardioprotective actions 
TABLE 2: Phytochemicals exhibiting cytoprotection in the in vitro models of DOX-induced cardiotoxicity.

\begin{tabular}{|c|c|c|c|c|}
\hline Phytochemical & $\begin{array}{c}\text { Concentration } \\
\text { of the } \\
\text { phytochemical }\end{array}$ & $\begin{array}{l}\text { Cell culture } \\
\text { model }\end{array}$ & $\begin{array}{c}\text { DOX dose and } \\
\text { time of } \\
\text { incubation }\end{array}$ & References \\
\hline Arjunolic acid & $100 \mu \mathrm{g} / \mathrm{mL}$ & $\begin{array}{l}\text { Neonatal rat } \\
\text { cardiomyocytes }\end{array}$ & $1 \mu \mathrm{M}$ for $12 \mathrm{~h}$ & [13] \\
\hline Apigenin & $25-100 \mu \mathrm{M}$ & $\begin{array}{c}\text { Rat heart } \\
\text { cardiomyocytes }\end{array}$ & $100 \mu \mathrm{M}$ for $8 \mathrm{~h}$ & {$[16]$} \\
\hline Avicularin & $10-80 \mu \mathrm{M}$ & H9c2 cells & $20 \mu \mathrm{M}$ for $24 \mathrm{~h}$ & {$[17]$} \\
\hline Berberine & $\begin{array}{c}0.06,0.25,1.0, \\
\text { and } 4.0 \mu \mathrm{M}\end{array}$ & $\begin{array}{c}\text { Neonatal rat } \\
\text { cardiomyocytes } \\
\text { and MCF-7 cells }\end{array}$ & $1 \mu \mathrm{M}$ for $2 \mathrm{~h}$ & {$[19]$} \\
\hline Baicalein & $25 \mu \mathrm{M}$ & $\begin{array}{c}\text { Chick embryo } \\
\text { cardiomyocytes } \\
\text { and MCF-7 cells }\end{array}$ & $\begin{array}{c}1,10,50, \text { or } \\
100 \mu \mathrm{M} \text { for } 24 \mathrm{~h}\end{array}$ & {$[22]$} \\
\hline Calceolarioside & $40 \mu \mathrm{M}$ & H9c2 cells & $\begin{array}{c}1,2, \text { or } 5 \mu \mathrm{M} \text { for } \\
30 \mathrm{~h}\end{array}$ & {$[27]$} \\
\hline 23-Hydroxybetulinic acid & $\begin{array}{c}0.2,2 \text {, and } \\
20 \mu \mathrm{M}\end{array}$ & H9c2 cells & $5 \mu \mathrm{M}$ for $18 \mathrm{~h}$ & {$[60]$} \\
\hline Isorhamnetin & $\begin{array}{c}3.125 \text { to } \\
25 \mu \mathrm{g} / \mathrm{mL} \\
\end{array}$ & $\begin{array}{c}\text { MCF-7, HepG2, } \\
\text { and Hep2 cells }\end{array}$ & $1 \mu \mathrm{M}$ for $36 \mathrm{~h}$ & {$[66]$} \\
\hline Kaempferol & 5 to $50 \mu \mathrm{M}$ & H9c2 cells & $1 \mu \mathrm{M}$ for $24 \mathrm{~h}$ & {$[69]$} \\
\hline Morin hydrate & $0.17 \mathrm{mM}$ & $\begin{array}{l}\text { ECV304 and } \\
\text { HepG2 cells }\end{array}$ & $6 \mathrm{mM}$ for $12 \mathrm{~h}$ & {$[77]$} \\
\hline Naringenin-7-O-glucoside & $10-80 \mu \mathrm{M}$ & H9c2 cells & $10 \mu \mathrm{M}$ for $24 \mathrm{~h}$ & {$[83]$} \\
\hline Osthole & $10-40 \mu \mathrm{M}$ & $\begin{array}{c}\text { Neonatal rat } \\
\text { cardiomyocytes }\end{array}$ & $1 \mu \mathrm{mol}$ for $24 \mathrm{~h}$ & {$[90]$} \\
\hline Luteolin-7-O- $\beta$-D-glucopyranoside & 5,10 , and $20 \mu \mathrm{M}$ & H9c2 cells & $20 \mu \mathrm{M}$ for $24 \mathrm{~h}$ & {$[75]$} \\
\hline Luteolin-7-O- $\beta$-D-glucopyranoside & $5-80 \mu \mathrm{M}$ & H9c2 cells & $10 \mu \mathrm{M}$ for $24 \mathrm{~h}$ & {$[76]$} \\
\hline Vincristine & $10-30 \mu \mathrm{M}$ & $\begin{array}{c}\text { Adult mouse } \\
\text { cardiomyocytes }\end{array}$ & $\begin{array}{c}15 \text { and } 20 \mu \mathrm{g} / \mathrm{mL} \\
\text { for } 24 \mathrm{~h}\end{array}$ & {$[144]$} \\
\hline Sulforaphane & $2.5 \mu \mathrm{M}$ & H9c2 cells & $\begin{array}{c}5 \mu \mathrm{g} / \mathrm{mL} \text { for } \\
16-18 \mathrm{~h}\end{array}$ & [129] \\
\hline C-Phycocyanin & $10 \mu \mathrm{M}$ & $\begin{array}{c}\text { Adult rat } \\
\text { cardiomyocytes }\end{array}$ & $\begin{array}{c}10 \mu \mathrm{M} \text { for } 4,24, \\
\text { and } 48 \mathrm{~h}\end{array}$ & {$[94]$} \\
\hline Plantainoside D & $1-20 \mu \mathrm{g} / \mathrm{mL}$ & H9c2 cells & $\begin{array}{l}1,2 \text {, and } 4 \mu \mathrm{M} \\
\text { for } 30 \mathrm{~h}\end{array}$ & {$[93]$} \\
\hline Sesamol & $12.5-50 \mu \mathrm{M}$ & H9c2 cells & $1 \mu \mathrm{M}$ for $30 \mathrm{~min}$ & {$[125]$} \\
\hline Tetrahydroxystilbene glucoside & $3-300 \mu \mathrm{M}$ & $\begin{array}{c}\text { Neonatal rat } \\
\text { cardiomyocytes }\end{array}$ & $\begin{array}{c}1 \mu \mathrm{mol} / \mathrm{L} \text { for } \\
24 \mathrm{~h}\end{array}$ & {$[143]$} \\
\hline Chrysoeriol & $20 \mu \mathrm{g} / \mathrm{mL}$ & H9c2 cells & $1 \mu \mathrm{M}$ for $24 \mathrm{~h}$ & {$[43]$} \\
\hline Visnagin & $20 \mu \mathrm{M}$ & $\begin{array}{l}\text { Neonatal rat and } \\
\text { zebrafish } \\
\text { cardiomyocytes, } \\
\text { cardiac HL-1 } \\
\text { cells }\end{array}$ & $100 \mu \mathrm{M}$ for $48 \mathrm{~h}$ & {$[146]$} \\
\hline Z-Guggulsterone & $1-30 \mu \mathrm{M}$ & H9C2 cells & $1 \mu \mathrm{M}$ for $24 \mathrm{~h}$ & {$[143]$} \\
\hline Tanshinone IIA & $\begin{array}{c}0.1,0.3,1 \text {, and } \\
3 \mu \mathrm{M}\end{array}$ & $\begin{array}{c}\text { Neonatal rat } \\
\text { cardiomyocytes }\end{array}$ & $1 \mu \mathrm{M}$ for $24 \mathrm{~h}$ & {$[134]$} \\
\hline Tanshinone IIA & $1.6-40 \mu \mathrm{M}$ & H9c2 cells & $1 \mu \mathrm{M}$ for $24 \mathrm{~h}$ & {$[133]$} \\
\hline Tanshinone IIA & $\begin{array}{l}0.5,1 \text {, and } \\
2 \mu \mathrm{mol} / \mathrm{L}\end{array}$ & $\begin{array}{c}\text { Neonatal rat } \\
\text { cardiomyocytes }\end{array}$ & $\begin{array}{c}1 \mu \mathrm{mol} / \mathrm{L} \text { for } \\
24 \mathrm{~h}\end{array}$ & {$[132]$} \\
\hline Sodium tanshinone IIA sulphonate & $0.05-0.5 \mathrm{mM}$ & $\begin{array}{c}\text { Mice heart } \\
\text { mitochondria }\end{array}$ & $\begin{array}{c}0.2 \mathrm{mmol} \text { for } \\
10 \mathrm{~min}\end{array}$ & {$[131]$} \\
\hline Anthocyanidins and anthocyanins & $0-100 \mu \mathrm{M}$ & $\begin{array}{l}\text { H9c2 cells and } \\
\text { MCF-7 cells }\end{array}$ & $1 \mu \mathrm{M}$ for $24 \mathrm{~h}$ & {$[14]$} \\
\hline
\end{tabular}


TABLE 2: Continued.

\begin{tabular}{lcccc}
\hline Phytochemical & $\begin{array}{c}\text { Concentration } \\
\text { of the } \\
\text { phytochemical }\end{array}$ & $\begin{array}{c}\text { Cell culture } \\
\text { model }\end{array}$ & $\begin{array}{c}\text { DOX dose and } \\
\text { time of } \\
\text { incubation }\end{array}$ \\
\hline $\begin{array}{l}\text { Caffeic, chlorogenic, and rosmarinic } \\
\text { acid }\end{array}$ & 100 and $200 \mu \mathrm{M}$ & $\begin{array}{c}\text { Rat heart } \\
\text { microsomes and } \\
\text { mitochondria }\end{array}$ & $100 \mu \mathrm{M}$ for $8 \mathrm{~h}$ & References \\
\hline
\end{tabular}

ECV304 cells: human umbilical vein endothelial cells; HepG2 cells: human hepatocellular carcinoma cells; MCF-7 cells: human breast carcinoma; H9c2 cells: rat ventricular cardiomyoblast cells.

[43]. Chrysoeriol has been found to reduce cell death and attenuate ROS generated oxidative stress and lipid peroxidation in DOX-induced cardiotoxicity in H9c2 cardiomyoblasts without affecting antitumor activity of DOX [43]. For an evidence based approach, additional studies on its cardioprotective efficacies are warranted.

2.14. Curcumin. Curcumin is a phenolic yellow pigment constituent found in the rhizomatous parts of Curcuma longa (turmeric) [44]. Several in vitro and in vivo studies have demonstrated curcumin cardioprotective actions against DOX-induced myocardial toxicity. The key mechanisms postulated for curcumin cardioprotective activity include diminution of oxidative stress, inflammation, and associated cell death pathways [45-48]. Although curcumin has been reported to elicit several beneficial effects in various preclinical studies, the bioavailability is yet to be established in human subjects. Therefore, derivatives of curcumin are being perused to increase its bioavailability and, in this direction, a recent report suggests that the nanoparticle of curcumin could ameliorate DOX-induced cardiotoxicity [49].

2.15. Eugenol. Eugenol is the active component of essential oil isolated from Syzygium aromaticum, popularly known as clove, which is one of the common ingredients of spice mixtures [50]. Eugenol treatment was shown to significantly improve antioxidant defense mechanisms, decrease lipid peroxidation, and attenuate abnormal $\mathrm{Ca}^{2+}$ transients in the cardiomyocytes along with inhibition of apoptosis in rat hearts following acute DOX administration. Eugenol also preserved the myocardium and restored hemodynamics along with preserved histology [51].

2.16. Frederine. 7-Monohydroxyethylrutoside (monoHER2, frederine) is a synthetic flavonoid, and it significantly inhibited DOX-induced myocardial toxicity, via suppression of oxidative stress and apoptosis in a chronic murine model $[52,53]$. In addition, monoHER2 did not interfere with DOX anticancer activity in vitro and in vivo [54]. Considering these observations, monoHER 2 could be further developed for this clinical application as cardioprotective adjuvant.

2.17. Gingerol. Gingerol is the pungent phenolic constituent of Zingiber officinalis (ginger) [55]. In a chronic model of
DOX-induced cardiomyopathy, gingerol inhibited DOXinduced myocardial ROS generation, inflammation via attenuation of NF- $\kappa \mathrm{B}$ activation, and downregulation of soluble receptor for advanced glycation end products (sRAGE). In addition, gingerol also inhibited myocardial apoptosis via mitigating caspase-3 activities [56].

2.18. Ginsenosides. Ginsenosides are the saponin constituents of Panax notoginseng, popularly known as ginseng in traditional Chinese medicine [57]. Ginsenosides have been classified into protopanaxatriols (Rgl, Rhl, and PPT) and protopanaxadiols ( $\mathrm{Rg} 3, \mathrm{Rh} 2$, and $\mathrm{PPD}$ ). Of the ginsenosides, protopanaxadiols such as ginsenoside Rb1, ginsenoside $\mathrm{Rh} 2$, and compound $\mathrm{K}$ have been shown to exhibit anticancer and anti-inflammatory activities [57].

Ginsenoside Rh2 (Rh2) has been shown to elicit cardioprotective effects against DOX-induced cardiotoxicity in $\mathrm{H} 9 \mathrm{c} 2$ cell line, as well as in vivo in an acute mouse and chronic rat model of DOX-induced cardiomyopathy. Rh2 enhanced cell viability of $\mathrm{H} 9 \mathrm{c} 2$ cells and ameliorated DOXinduced release of the CK-MB, LDH. Furthermore, Rh2 ameliorated DOX-induced myocardial toxicity in mouse and rats via suppressing oxidative stress and improved the indices of cardiac function as determined by ECG [58].

2.19. Diosgenin. Diosgenin is a steroid saponin found abundantly in several plants including Solanum and Dioscorea species and Costus speciosus. In a chronic model of DOXinduced cardiomyopathy, diosgenin elicited cardioprotective effects, via activation of prosurvival kinase, protein kinase A (PKA), diminution of p38-MAPK, caspase-3 activities, and generation of free radicals along with attenuation of inflammatory mediators. Mechanistically, it was found to improve myocardial fibrosis and increase the cardiac levels of cGMP via modulation of phosphodiesterase-5 activity [59].

2.20. Hydroxybetulinic Acid. 23-Hydroxybetulinic acid is isolated from Pulsatilla chinensis. In a chronic murine model of DOX-induced cardiomyopathy, 23-hydroxybetulinic acid significantly improved the survival of the animals and inhibited apoptosis mainly via inhibition of DOX metabolism in the mitochondria. Similar results were also obtained in H9c2 cells [60].

2.21. Hesperidin. Hesperidin is a bioflavonoid abundantly found in vegetables and citrus fruits such as oranges, lemons, 
and grapefruits [61]. Citrus flavonoids have been shown to reduce risk of cardiovascular diseases prominently due to their antioxidant and anti-inflammatory effects involving numerous cell signaling pathways [61]. It has been found to improve antioxidant status, inhibit lipid peroxidation, and reduce myocardial enzyme leakage by salvaging myocardium in DOX-induced cardiotoxicity in acute toxicity model conducted rats [62]. Additionally, it sensitized cancer cells to DOX-induced apoptosis and showed synergism in inhibiting P-gp and multidrug resistance, thus appearing to be effective as an adjunct to enhance the efficacy and attenuate the resistance to DOX during chemotherapy [63]. Thus, the potential of citrus flavonoids as cochemotherapeutic and cardioprotective agents is encouraging but further studies are warranted for conclusive evidence in cancer as well as chemotherapy associated cardiotoxicity. In addition, hesperetin (aglycone) derivative of hesperidin also ameliorated chronic DOX treatment associated cardiotoxicity in rats, via attenuation of p38-MAPK, caspase-3, and NF- $\kappa$ B activation and oxidative stress in the myocardial tissues [64].

2.22. Hydroxytyrosol. Hydroxytyrosol is a polyphenolic constituent in Olea europaea, popularly known as olive oil, which is widely used in food and medicine [65]. Hydroxytyrosol has been shown to improve cardiac function by maintaining homeostasis at mitochondrial level, by preserving mitochondrial electron transport chain complexes I-IV and inhibiting apoptosis-inducing factor, and oxidative stress markers in chronic DOX-induced cardiotoxicity in rats harboring breast cancer [65]. Furthermore, hydroxytyrosol did not compromise the DOX antitumor activity against the implanted tumor cells and also improved the survival of the animals [65].

2.23. Isorhamnetin. Isorhamnetin is a flavonol aglycone abundantly found in several medicinal plants, such as Hippophae rhamnoides (sea buckthorn) [66]. Isorhamnetin significantly conferred cardioprotection in a chronic model of DOX-induced cardiotoxicity. The mechanism of cardioprotection involves suppression of oxidative stress and activation of mitochondrial apoptotic pathway and mitogen-activated protein kinase pathways, suggesting antioxidant mediated cardioprotective mechanism. Similar results were obtained in H9c2 cells [66]. Furthermore, it also synergistically improved the DOX anticancer activity in tumor cell lines [66].

2.24. Indole-3-carbinol. Indole-3-carbinol is a natural indole compound predominantly found in cruciferous vegetables [67]. In a chronic DOX-infusion associated cardiotoxicity murine model, indole-3-carbinol was found to reduce solid Ehrlich tumor size and volume, augment antioxidant defense, and inhibit lipid peroxidation, leading to stabilization of cell membrane and reduced leakage of myocyte injury marker enzymes. It was also found to decrease sphingosine kinase 1 (SphK1) activity and inflammatory mediators along with mitigating histological perturbations and modulating cell death mediators [67].
2.25. Kaempferol. Kaempferol is one of the most common dietary flavonoids and it is well studied for its antiapoptotic, cardioprotective, antioxidative, anti-inflammatory, chemopreventive, and anticancer properties as well as modulation of chemoresistance [68]. The cardioprotective effects of kaempferol against DOX-induced cardiotoxicity in rats were demonstrated using a chronic model. Kaempferol counteracted cardiotoxicity by inhibiting p53 expression in mitochondrion-dependent apoptotic signaling and ERKdependent mitogen-activated protein kinase pathway following binding to the promoter region of the Bax proapoptotic gene. It also effectively suppressed DOX-induced extracellular signal-regulated kinase (ERK1/2) activation but had no effect on p38 and JNK [69].

2.26. Lycopene. Lycopene is a carotenoid and nonprovitamin A found abundantly in Lycopersicum esculentum (tomatoes) and known to impart color to tomatoes [33]. Karimi et al., in a very early study, demonstrated the protective effect of tomato extract and lycopene on acute DOX-induced cardiotoxicity in mice [70]. Tomato extract and lycopene prevented rise in myocyte injury marker enzyme, CK-MB, in serum and ameliorated cardiomyocytes injury evidenced by histopathological examination. Furthermore, Yilmaz et al. studied the protective role of lycopene in DOX-induced heart and kidney toxicities using biochemical and histopathological assessments and reported that lycopene has potential to inhibit lipid peroxidation and improve antioxidants evidenced by reduced lipid peroxides and improved GSH in both the heart and the kidneys. The protective effect was further substantiated by histopathological changes. The authors concluded that treatment with lycopene might prevent cardiac and renal toxicities in rats [71]. However, the results were not further substantiated by functional improvement as lycopene did not prevent left ventricular systolic dysfunction induced by DOX [72]. However, it suppressed DOX-induced myocyte damage without preventing interstitial collagen accumulation increase [72].

Lycopene supplementation also increased lycopene absorption in heart, liver, and plasma and, in another study, the same group of authors showed that there was no depletion of lycopene from myocardium of lycopene-supplemented rats treated with DOX and that higher antioxidant capacity in myocardium and less oxidative cleavage of lycopene in intestinal mucosa of DOX-treated rats suggest an antioxidant role of DOX rather than acting as a prooxidant [73]. The authors further showed that tomato-oleoresin enhances the chemotherapeutic effect of DOX. It maintained lycopene levels in heart and protected against cardiac oxidative DNA damage induced by DOX in rats [73]. The lycopene protected the heart against DOX associated cardiotoxicity by several mechanisms including the quenching of singlet oxygen, peroxy radicals, reaction with free radicals, restoring levels of vitamin $\mathrm{E}$ and vitamin $\mathrm{C}$, reducing DNA damage, restoring cellular antioxidants, and preventing depletion of glutathione. Several studies showed the potential role of lycopene in the prevention of side effects of antineoplastic drugs in cell culture and animal models [74]. These results 
suggest that tomato extract and lycopene inhibit DOX cardiotoxicity and collectively might serve as a novel combination chemotherapeutic agent with DOX to limit free radical-mediated organ injury. However, further studies are required to investigate the role of lycopene in mitigating the side effects of chemotherapy in human subjects.

2.27. Luteolin-7-O- $\beta$-D-glucopyranoside. Luteolin-7-O- $\beta$-Dglucopyranoside is a flavonoid isolated from the plant Dracocephalum tanguticum that is widely used in Chinese and Tibetan traditional medicine [75]. The cytoprotective activities were demonstrated on DOX-induced cytotoxicity in H9c2 cardiomyocytes. Among several isolated compounds, luteolin-7-O- $\beta$-D-glucopyranoside was found to show antioxidant effect and a potent cytoprotective activity against DOX-induced toxicity as evidenced by decreased death of $\mathrm{H} 9 \mathrm{c} 2$ cells, reduced myocyte injury marker enzymes, and reduced intracellular concentration of ROS and $\mathrm{Ca}^{2+}$ [75]. Recently, Yao et al. also demonstrated protective effects of luteolin-7-O-glucoside on DOX cytotoxicity in H9c2 cells [76]. It was found to improve cell viability and ameliorate ROS generation and mitochondrial depolarization. Furthermore, it enhanced the expression of prosurvival kinases and diminished ROS generation [76].

2.28. Morin Hydrate. Morin hydrate is a biflavonoid commonly found in fruits such as guava, fig, almond, grapes, and apple and vegetables such as onion, seed weeds, and several other members of Moraceae family [77]. It has been observed to enhance antioxidant defense against oxidative stress in human umbilical vein endothelial cells (ECV304) and HepG2 cells and minimize DOX toxicity in ECV304 and primary mouse cardiomyocytes [77]. However, in vivo studies regarding morin efficacy in combating DOX-induced cardiotoxicity are still lacking.

2.29. Mangiferin. Mangiferin is a xanthonoid structure with C-glucosyl linkage and polyhydroxy component found in many plant species; however, Mangifera indica (mango tree) is the major source [78]. Using a chronic model of cardiomyopathy in rats, mangiferin has been shown to exert cardioprotective action against DOX-induced cardiotoxicity by inhibition of proinflammatory mediators and proapoptotic genes and regulating calcium homeostasis modulating proteins [79].

2.30. Naringin. Naringin is a flavanone glycoside abundantly found in citrus fruits such as lemon, oranges, and grapefruits and in tomatoes. It has been documented to possess numerous biological properties such as antioxidant, antiinflammatory, and antiapoptotic activities [80]. In an acute model of DOX-induced cardiotoxicity, naringin treatment improved antioxidant defense and inhibited lipid peroxidation along with histopathological preservation, thereby reducing leakage of myocyte marker enzymes. It also decreased the levels of inflammatory mediators and restored the mitochondrial complexes (I-IV) activities in the heart tissues along with histopathological salvage [80]. The studies indicate cardioprotective effects; however, further clinical research is required to provide significant insights into the mechanisms underlying the effects of naringin on human subjects.

2.31. Naringenin and Its Derivatives. Naringenin is a flavanone commonly found in citrus fruits such as grapefruit, orange, and lemon [80]. Arafa et al. demonstrated that naringenin elicited antioxidant mediated protection against DOX-induced cardiac toxicity in Swiss albino rats [81]. Recently, the combination of p-coumaric acid and naringenin was found to be superior in exerting antioxidant mediated cardioprotection against DOX-induced cardiotoxicity in rats [82]. Additionally, naringenin enhanced antitumor effect of DOX by selectively modulating drug efflux pathways; that is, it inhibited the activity of multidrug resistance-associated protein and did not affect the in vivo pharmacokinetics of intravenously administered DOX [80].

Naringenin-7-O-glucoside is a flavanone glycoside isolated from Dracocephalum rupestre. It has been demonstrated to protect against DOX-induced cardiotoxicity in $\mathrm{H} 9 \mathrm{c} 2$ cells [83]. Particularly, naringenin-7-O-glucoside improved cell viability, prevented the release of myocyte injury marker enzymes LDH and CK, and augmented antioxidant defense [84]. Furthermore, naringenin-7-O-glucoside was observed to enhance $\mathrm{NAD}(\mathrm{P}) \mathrm{H}$ : quinone oxidoreductase (NQO1) and ERK activation and Nrf2 protein levels in DOX stressed H9c2 cells. These phenotypic changes brought about by naringenin-7-O-glucoside are attributed to the induction of antioxidant defense and attenuation of cell death pathways [85]. However, in vivo studies are yet to be performed.

2.32. Ocotillol. Ocotillol is an aglycone derivative of pseudoginsenoside-F11, which is devoid of sugar moiety and is found in American ginseng, Panax quinquefolius. $\mathrm{Fu}$ et al. reported that ocotillol enhances survival rate of animals in both acute and chronic models of DOXinduced cardiotoxicity. Ocotillol prevented depletion of glutathione and lipid peroxidation along with restoration of myocyte injury marker enzymes following preservation of cardiomyocytes cell membrane. Furthermore, ocotillol also improved cardiac function and hence was suggested as an adjuvant for counteracting DOX-induced cardiotoxicity [86].

2.33. Oleuropein. Oleuropein is a phenolic constituent of Olea europaea (olive oil) [87]. Andreadou et al. have shown the protective effect of oleuropein against DOX-induced acute cardiotoxicity in rats. Oleuropein was found to restore the myocardial necrosis marker enzyme levels and attenuation of oxidative stress and apoptosis [88]. Moreover, the same group of authors in a separate study demonstrated that oleuropein treatment aids the compensation of distressed energy metabolic pathways mechanistically by restoration of metabolites to the normal levels as DOX generated free radicals nonenzymatically convert pyruvate to acetate and alpha-ketoglutarate to succinate [87]. The cardioprotective role of oleuropein in chronic DOX-induced 
cardiomyopathy has also been demonstrated [89]. Particularly, oleuropein treatment significantly suppressed DOXinduced oxidative/nitrative stress, augmented prosurvival kinases, and improved the cardiac functions [89].

2.34. Osthole. Osthole is a coumarin compound found in several medicinal plants such as Cnidium monnieri and Angelica pubescens [90]. In rat neonatal cardiomyocytes, osthole significantly improved the survival of the cells by abrogating apoptosis, wherein the mechanism appeared to involve the suppression of mitochondrial pathway of apoptosis triggered by DOX [90]. However, in vivo studies have not been conducted, and it is warranted to recapitulate the in vitro cardioprotective actions of osthole.

2.35. p-coumaric Acid. p-coumaric acid is a phenolic acid that serves as a precursor of other phenolic compounds and is found in plants such as peanut, tea, and coffee [82]. pcoumaric acid has been shown to attenuate oxidative stress and inhibit myocyte injury in DOX-induced myocardial injury in rats [91]. In a separate study, p-coumaric acid in combination with naringenin showed cardioprotection by augmentation of antioxidant defense against DOX-induced cardiotoxicity in rats [82].

2.36. Periplogenin. Periplogenin is a cardenolide isolated from Aegle marmelos, commonly known as Bael in the traditional Indian system of medicine [92]. Periplogenin has been shown to decrease lipid peroxide levels, improve antioxidant defense, and salvage cardiomyocytes in a chronic model of DOX-induced cardiotoxicity in rats [92].

2.37. Plantainoside D. Plantainoside D is an iridoid glucoside isolated from Picrorhiza scrophulariflora (Picrorhiza). The chemopreventive and antioxidant activities encouraged evaluating the cardioprotective effect of plantainoside $\mathrm{D}$ against DOX-induced apoptosis in H9c2 cells. Plantainoside $\mathrm{D}$ was found to inhibit oxidative stress and proinflammatory cytokines expression and attenuate apoptosis in H9c2 cardiomyoblasts [93].

2.38. Phycocyanin. C-Phycocyanin is a biliprotein found in Spirulina platensis, blue-green algae. Phycocyanin has been found to protect against DOX-induced oxidative stress and apoptosis in adult rat cardiomyocytes as evidenced by reduced ROS formation, DNA fragmentation, and attenuation of Bax as well as release of cytochrome $\mathrm{C}$ and increase in the activity of caspase-3 [94]. However, these in vitro findings are yet to be confirmed in rodent model of DOX-induced cardiomyopathy.

2.39. Proanthocyanidin and Derivatives. Proanthocyanidins are a mixture of structurally and functionally diverse chemicals which are predominantly found in grape seed and show high bioavailability and protect the organs from toxic chemicals used to induce diseases in in vitro and in vivo studies [95, 96]. Ray et al. reported the bioavailability and protective property of grape seed proanthocyanidin and a novel IH636 grape seed proanthocyanidin extract against DOX-induced cardiotoxicity as well as multiorgan protection in mice [97]. Bagchi et al. further reported that IH636 proanthocyanidin extract afforded protection was superior to vitamin $\mathrm{C}$, vitamin $\mathrm{E}$, and beta-carotene and demonstrated significant cytotoxicity towards human breast, lung, and gastric adenocarcinoma cells, while enhancing the growth and viability of normal cells in both in vitro and in vivo studies [98]. In another study, proanthocyanidins enhanced DOX-induced antitumor effect and reversed drug resistance and mechanisms attributed partially to the promotion of DOX-induced apoptosis through elevation of intracellular DOX, $\mathrm{Ca}^{2+}$, and $\mathrm{Mg}^{2+}$ concentration and reduction of $\mathrm{pH}$ value and mitochondrial membrane potential in DOX-resistant K562/DOX cells [99]. Furthermore, proanthocyanidin strongly enhanced the antitumor activity of DOX and ameliorated chronic DOX-induced myocardial oxidative stress and immunosuppression in tumor-bearing mice [99]. In addition, grape seed proanthocyanidin also showed antioxidant mediated cardioprotection against both high and low dose DOX-induced cumulative chronic cardiotoxicities in rats [100].

2.40. Resveratrol. Resveratrol, a natural phytoalexin, is commonly found in Vitis vinifera (grapes) [101]. Resveratrol induced antioxidants and phase 2 enzymes in the $\mathrm{H} 9 \mathrm{c} 2$ cells, accompanied by increased resistance to oxidative and electrophilic cell injury [102]. Additionally, there was no significant effect of resveratrol on NADPH-cytochrome P450 reductase (P-450 reductase), which plays an important role in the metabolism of many endogenous compounds and xenobiotics including DOX. The enzyme P-450 reductase activates them to their more toxic metabolites via one electron reduction which triggers free radical cascade. In some cases, however, such transformation is essential to produce therapeutic effect of anticancer drugs [42].

In DOX-induced cardiotoxicity in rats, resveratrol has been shown to ameliorate the severity of cardiac dysfunction and prevent oxidant stress responses [103, 104]. Furthermore, resveratrol was found to confer cardioprotection and reduce cardiac fibrosis in acute as well as chronic in vivo models of DOX-induced cardiomyopathy in rats. Mechanistically, resveratrol has been demonstrated to protect against DOXinduced oxidative stress through changes in mitochondrial function, specifically the Sirtl pathway, leading to cardiac cell survival [105]. Resveratrol attenuated DOX-induced cardiomyocyte apoptosis in mice via upregulation of Sirtlmediated p53 deacetylation and activation of Sirtl, a NAD ${ }^{+}$dependent deacetylase, resulting in improved mitochondrial function, which culminates in activation of the transcription factors which coordinate expression of key antioxidant proteins by binding to the antioxidant response elements that regulate cell survival [106]. The overexpression of Sirtl inhibited cell apoptosis by suppression of p38-MAPK phosphorylation and caspase- 3 activation along with amelioration of ROS generation and prevented DOX-induced functional loss in DOX-induced cardiomyocyte injury [106]. DOX induces autophagy in cardiomyocytes which is a degradation system 
for eukaryotic cells to turn over organelles and long-lived proteins, thereby maintaining cellular homeostasis. Thus, aberrant autophagy activity impairs basal cardiac structure and function, making animals more sensitive to stressinduced heart failure. The ability of resveratrol to inhibit autophagy is mediated by inhibition of p70S6 kinase 1 (S6 K1) that is essential for resveratrol to suppress DOX-induced autophagy and cytotoxic effects [107].

DOX inhibits AMP-activated protein kinase (AMPK), resulting in Sirtl dysfunction and p53 accumulation in mouse embryonic fibroblasts, and pharmacological activation of AMPK by resveratrol has been shown to alleviate the side effects of DOX in H9c2 cells [108]. Furthermore, resveratrol has been shown to confer cardioprotection in DOXinduced cardiomyocyte apoptosis in nude mice by induction of heme oxygenase-1 (HO-1) mediated mechanisms [109, 110]. Resveratrol was also reported to aid the differentiation of adipose-derived mesenchymal stem cells to cardiomyocytes and protected against noxious effects of DOX to the myocardium [111]. Furthermore, resveratrol supplement along with exercise training was found to be more effective in preventing DOX-induced LV remodeling associated with the reduction of DOX-induced oxidative stress [112]. In spite of these advances made in preclinical studies, resveratrol bioavailability is seldom established in human subjects and this warrants further approaches to extend its beneficial effects to mankind [113].

2.41. Robinin. Robinin is a flavonoid glycoside isolated from leaves of Vigna unguiculata, a dietary plant used in traditional cuisine in India [114]. Treatment with robinin was found to improve endogenous antioxidants, reduce ROS generation, and inhibit lipid peroxidation and proinflammatory mediators such as cyclooxygenase (COX2) and lipoxygenase (LOX15) along with restoring myocyte injury marker enzymes. The improvement in the level of transforming growth factor- $\beta 1$ (TGF- $\beta 1$ ), Smad2, murine double minute (Mdm2), Smad3, cyclin-dependent kinase inhibitor $2 \mathrm{~A}$, Smad4, and Smad7 in addition to favorable modulation of p53, Bcl-2, and Bax revealed the cardioprotective mechanism of robinin in combating DOX-induced cardiotoxicity [114].

2.42. Rosmarinic Acid. Rosmarinic acid is an ester of caffeic acid abundantly found in numerous plants, being most common in Boraginaceae and Lamiaceae families [115]. Rosmarinic acid showed remarkable cytoprotection against DOX toxicity in neonatal rat cardiomyocytes and DOX-induced lipid peroxidation of heart membranes, mitochondria, and microsomes and effects were found to be comparable to dexrazoxane [116]. Furthermore, it inhibited DOX-induced oxidative stress and apoptosis in $\mathrm{H} 9 \mathrm{c} 2$ cardiomyoblasts by improving cell viability, inhibiting the production of ROS, and activation of prosurvival kinases [115]. However, in vivo cardioprotective actions against DOX-induced cardiotoxicity are hitherto unknown.
2.43. Salvianolic Acids. Salvianolic acids especially salvianolic acid A and salvianolic acid B are the most abundant watersoluble compounds extracted from Salvia miltiorrhiza (Danshen or red sage) [117]. Salvianolic acid A has been shown to protect against DOX-induced mitochondrial toxicity in vitro in rat cardiomyocytes due to its antioxidant action, without antagonizing effect on the antitumor activity of DOX [118]. The protective effects of salvianolic acid were reconfirmed in vivo in another study, against DOX cardiotoxicity in mice [117], via abrogation of oxidative stress and inflammation.

2.44. Schisandrin B. Schisandrin B, a dibenzocyclooctadiene lignin, is isolated from the fruit of Schisandra chinensis. It has been shown to salvage cardiomyocytes, via conferring antioxidant defense by restoring glutathione flux in an acute animal model of DOX-induced cardiotoxicity [119]. Furthermore, it also mitigated DOX-induced cardiotoxicity in rabbits [120]. Recently, cardioprotective effects of schisandrin $B$ against DOX-induced cardiotoxicity were reconfirmed and the mechanism of protection was evidenced by amelioration of proinflammatory cytokines, lipid peroxidation, DNA damage, apoptosis, and MAPK activation in the myocardial tissues [121, 122].

2.45. Salidroside. Salidroside, a phenylethanoid glycoside, has been isolated from the roots of Rhodiola rosea (Roseroot). Wang et al. demonstrated that treatment of salidroside to either $\mathrm{H} 9 \mathrm{c} 2$ cells or mice stressed with acute DOX administration conferred cardioprotective effects. The mechanism was defined to involve antioxidant and suppression of proapoptotic mediators [123]. The cardioprotective effects of salidroside were reconfirmed in a placebo controlled clinical trial wherein sixty patients with breast cancer receiving epirubicin were given salidroside $(600 \mathrm{mg} /$ day $)$ or placebo starting 1 week before chemotherapy and assessed at baseline and 7 days after each new epirubicin dose of $100 \mathrm{mg} / \mathrm{m}^{2}$. Decline in strain rate peak was observed at an epirubicin dose of $200 \mathrm{mg} / \mathrm{m}^{2}$, with no significant differences between salidroside and placebo. At increasing cumulative doses of epirubicin, the strain rate normalized only with salidroside, showing a significant difference in comparison with placebo at epirubicin doses of $300 \mathrm{mg} / \mathrm{m}^{2}$. The authors concluded that salidroside may provide protection against chemotherapyinduced early left ventricular regional systolic dysfunction in patients with breast cancer. Based on preclinical and clinical data, salidroside needs to be investigated further in a larger population for advocating salidroside as an adjuvant to thwart the DOX-induced cardiotoxicity.

2.46. Sesamin. Sesamin is a major lignin obtained from seeds of Sesamum indicum. Sesamin was observed to increase the endogenous antioxidant enzymes and prevent mitochondrial damage via activation of Sirtl in an acute model of DOXinduced cardiotoxicity [124].

2.47. Sesamol. Sesamol is a phenolic constituent of oil obtained from seeds of Sesamum indicum and is used commonly as an edible oil. The cardioprotective effects of 
sesamol were confirmed in an in vivo study, wherein sesamol mitigated cumulative DOX-induced cardiomyopathy in rats [125]. Sesamol improved antioxidant defense status, reduced myocyte injury marker enzymes released from cardiomyocytes, and inhibited lipid hydroperoxide. The salvage of tissues evidenced by biochemical and histopathological studies demonstrated cardioprotective effects of sesamol [125]. However, further mechanistic studies should be carried out investigating the effect on DOX efficacy and pharmacokinetic interaction.

2.48. Silibinin. Silibinin, a flavonolignan, is an active component of Silybum marianum (milk thistle), popularly known as silymarin and known to constitute $50-70 \%$ of the silymarin extract. The cardioprotective effect exerted by silymarin, silibinin, dehydrosilibinin, and silychristin was comparable to that of dexrazoxane, while silydianin exerted the best protective effect [126].

2.49. Sulforaphane. Sulforaphane is an organosulfur compound found in a significant amount in cruciferous vegetables, especially in broccoli (Brassica oleracea) [127]. The cardioprotective effects of sulforaphane were first demonstrated in $\mathrm{H} 9 \mathrm{c} 2$ rat myoblasts as evidenced by reduced number of apoptotic cells along with decreased expression of proapoptotic proteins such as Bax, caspase-3, and cytochrome $\mathrm{C}$ [128]. It also reduced ROS generation and restored mitochondrial membrane potential [128]. Moreover, the cardioprotective effects of sulforaphane were found to be mediated by the activation of the Kelch-like ECH-associated protein 1 (Keap1)/NF-E2-related factor-2 (Nrf2)/antioxidantresponsive element (ARE) pathway, which in turn mediates the induction of HO-1 [129].

2.50. Tanshinone IIA and Derivatives. Tanshinones are the group of bioactive compounds isolated from Salvia miltiorrhiza (Danshen), a Chinese medicinal plant reputed for the management of cardiovascular diseases in particular, angina pectoris, atherosclerosis, myocardial infarction, and ischemic-reperfusion injury [130]. Sodium tanshinone IIA sulfonate, a water-soluble derivative of tanshinone IIA, was demonstrated to be beneficial in reducing DOX-induced cardiotoxicity in mice hearts and in cultured cardiomyocytes [131]. Treatment with sodium tanshinone IIA sulfonate prevented decrease in body weight and reduced myocardial lipid peroxidation in mice along with improved activities of endogenous antioxidant enzymes. In addition, the antioxidative mechanism was also supported by in vitro experiments showing that sodium tanshinone IIA sulphonate scavenged DOX semiquinone free radical in heart homogenate and inhibited DOX-induced mitochondrial lipid peroxidation and swelling [131].

Furthermore, another study demonstrated the beneficial effect of tanshinone IIA on decreasing DOX-induced apoptosis in neonatal rat cardiomyocytes and underlying molecular mechanisms [132]. Tanshinone IIA ameliorated apoptosis and ROS generation induced by DOX in a dosedependent manner. It was further supported by the inhibition of DOX-mediated reduction of the ratio of $\mathrm{Bcl}-2 / \mathrm{Bax}$ [132]. Furthermore, a separate study also recapitulated that tanshinone IIA significantly inhibited DOX-induced toxic effects in $\mathrm{H} 9 \mathrm{c} 2$ cells as well as in animal models of cardiotoxicity [133]. In this study, tanshinone IIA was shown to improve cell viability and ameliorate apoptosis of DOX-induced cytotoxicity in H9c2 cells. Furthermore, the cardioprotective effects of tanshinone IIA sodium sulfonate were confirmed by decreased ST interval and QRS interval in ECG; improved histological appearance of myocardium; increased myocardial tensile strength; and decreased fibrosis [133]. Recently, Hong et al. evaluated the protective effect of tanshinone IIA on DOX-induced cardiomyocyte apoptosis and explored its intracellular mechanisms using primary cultured neonatal rat cardiomyocytes. Tanshinone IIA was found to inhibit DOX-induced reactive oxygen species generation, reduce the expression of caspase-3 and cytochrome C, and increase $\mathrm{BcL}-\mathrm{x}(\mathrm{L})$ expression, resulting in protecting cardiomyocytes from DOX-induced apoptosis. In addition, tanshinone IIA also enhanced Akt phosphorylation in cardiomyocytes and inhibited apoptosis [134].

2.51. Tetrahydroxystilbene Glucoside. Tetrahydroxystilbene glucoside is one of the active components extracted from Polygonum multiflorum (knot grass). For the first time, Zhang et al. demonstrated its protective effect on neonate rat cardiomyocytes and on an acute mouse model of DOXinduced cardiotoxicity [135]. In the mouse model, it was shown to inhibit lipid peroxidation accompanying improved glutathione, reduced animal mortality, preserved histopathological changes, and restored levels of myocyte injury marker enzymes. In the in vitro study, it prevented DOX-induced loss of mitochondrial membrane potential, caspase- 3 activation, and upregulation of Bax protein expression along with upregulation of Bcl-2 and inhibited ROS generation. It was also observed to inhibit DOX-induced increases in intracellular $\mathrm{Ca}^{2+}$ and apoptosis of cardiomyocytes in a concentrationdependent manner [135].

2.52. Thymoquinone. Thymoquinone is the main active constituent of the volatile oil of Nigella sativa Linn., popularly known as black seed, used for culinary and medicinal purposes [136]. Thymoquinone suppressed DOX-induced cardiotoxicity in an acute murine model of cardiomyopathy, without compromising antitumor activity of DOX [137]. Furthermore, thymoquinone also circumvented DOX-mediated cardiotoxicity in acute model, wherein the key mechanism was postulated to involve antioxidant pathways [138]. Finally, thymoquinone synergistically increased DOX activity in several cancer cell lines and prevented DOX-induced toxicity in H9c2 cells [139].

2.53. Tetrandrine. Tetrandrine is a bisbenzylisoquinoline alkaloid isolated from the dried root of Stephania tetrandra. In a chronic model of DOX-induced cardiomyopathy, tetrandrine significantly inhibited myocardial apoptosis via quenching of ROS and restoration of mitochondrial capacity. These beneficial effects were corroborated with improved 
indices of cardiac function [140]. It is pertinent to note that tetrandrine had a negligible effect in DOX pharmacokinetics properties in rodents, suggesting that tetrandrine might be a suitable candidate to be developed as cardioprotective adjuvant [141].

2.54. Z-Guggulsterone. Guggulsterone is a major active component of Commiphora mukul, popularly known as Guggul and reputed for its antihyperlipidemic and cardioprotective effects in Ayurvedic medicine [142]. Wang et al. demonstrated the protective activity of guggulsterone against DOX-induced cytotoxicity in $\mathrm{H} 9 \mathrm{c} 2$ cells. It was found to improve cell viability, morphology, and cytotoxicity and cellular antioxidants along with inhibition of apoptosis by altering activity of PARP, caspase- 3 , cytochrome $\mathrm{C}$ release, and $\mathrm{Bcl}-2$ proteins and reducing the activation of NF- $\kappa \mathrm{B}$ [143].

2.55. Vincristine. Vincristine is an alkaloidal constituent isolated from Catharanthus roseus (Madagascar periwinkle), also known as Vinca rosea. Recently, its potential to prevent DOX-induced cardiomyocyte death and related mechanisms has been reported in adult mouse cardiac myocytes [144]. Vincristine treatment to cardiomyocytes in the presence of DOX increased the cell viability. This was concordant with decreased PARP and caspase- 3 activities and increased activation of prosurvival kinase Akt and diminished MAPK pathways [144]. However, the precise cardioprotective effects in vivo are yet to be demonstrated.

2.56. Visnagin. Visnagin is an active constituent isolated from fruit extracts of Ammi visnaga known as toothpick weed and used in traditional Chinese medicine for cardiovascular diseases [145]. Visnagin was recently shown to be cardioprotective in a zebrafish model of DOX-induced cardiomyopathy that recapitulates the cardiomyocyte apoptosis and contractility similar to those observed in humans [146]. Visnagin was found to rescue the cardiac performance and circulatory defects caused by DOX in zebrafish. It also attenuated DOX-induced apoptosis in cultured cardiomyocytes and in vivo in zebrafish and mouse hearts along with improved cardiac contractility in DOX-treated mice. Additionally, it did not interfere with DOX efficacy in several cultured tumor lines or in zebrafish and mouse xenograft models. Visnagin was observed to bind mitochondrial malate dehydrogenase $(\mathrm{MDH} 2)$, a key enzyme in the tricarboxylic acid cycle that contributed to cardioprotection [146].

\section{Concluding Remarks and Future Perspectives}

From the analysis of the literature, it is evident that several phytochemicals exhibited cardioprotective effects in vitro and in vivo against DOX-induced cardiotoxicity. The key pathways modulated by phytochemicals in cardiomyocytes include oxidative stress, inflammation, and cell death pathways, as demonstrated in Figure 1. Majority of the phytochemicals were demonstrated to elicit cardioprotective activity in preclinical studies. However, they have not been translated for clinical utility in human subjects. The major impediment to the development of phytochemical based cardioprotective adjuvants pertains to their negligible pharmacokinetic actions in human subjects. Particularly, the poor or lack of bioavailability in human subjects retards the enthusiasm for further pharmaceutical development [113, 147].

In order to improve the bioavailability of phytochemicals, various synthetic derivatives have been pursued [44]. Although significant strides have been taken in delineating the pathomechanisms for DOX-induced cardiotoxicity, still we do not have bona fide clinical biomarker to predict early changes in the myocardium of patients who received DOX treatment [148]. Therefore, it is of paramount significance to devise a biomarker to predict the DOX-induced cardiotoxicity, because most patients (cancer survivors) exhibit dilated cardiomyopathy several years after exposure to DOX [148]. Furthermore, from Table 1, it is evident that there is discrepancy regarding the employment of appropriate models in studying phytochemicals protective effects in nullifying DOX-induced cardiotoxicity. Therefore, future studies addressing the phytochemicals protective effects against DOX cardiotoxicity should utilize the physiologically relevant cumulative (chronic) dosage regimen in rodents. In addition, future studies should obligatorily investigate the noninterference of phytochemicals against DOX anticancer activities in orthotropic tumor-bearing mouse models.

In sum, to exploit the true potentials of plant-derived compounds for drug development, significant intellectual and financial contributions are warranted from academia and pharmaceutical industry. Unfortunately, the major impediment in this direction is the lack of proper intellectual property rights protection that could protect the financial viability of the drug development projects based on phytochemicals for the treatment of cardiomyopathy. This caveat coupled with other pharmacodynamics and pharmacokinetic lapses pertaining to the phytochemicals precludes the attention of major pharmaceutical companies in their portfolio investments toward the drug development. However, academic research should be directed to develop phytochemicals derived small molecules with significant bioavailability in human subjects. Perhaps this approach could be envisaged for translational application in combating DOX-induced cardiotoxicity.

\section{Competing Interests}

The authors disclose no competing interests.

\section{Authors' Contributions}

Shreesh Ojha, Hasan Al Taee, Sameer Goyal, and Umesh B. Mahajan researched the literature. Shreesh Ojha, Sameer Goyal, and Umesh B. Mahajan drafted the paper. Chandrgouda R. Patil and D. S. Arya edited the paper. Mohanraj Rajesh researched the literature and wrote and edited the paper. All authors read the contents of the paper and approved the same. 


\section{Acknowledgments}

Shreesh Ojha and Mohanraj Rajesh were supported by the intramural program grants sponsored by the College of Medicine and Health Sciences and Office of Research and Graduate Studies of the United Arab Emirates University.

\section{References}

[1] T. Simunek, M. Sterba, O. Popelova, M. Adamcova, R. Hrdina, and V. Gersl, "Anthracycline-induced cardiotoxicity: overview of studies examining the roles of oxidative stress and free cellular iron," Pharmacological Reports, vol. 61, no. 1, pp. 154171, 2009.

[2] N. Yamaguchi, T. Fujii, S. Aoi, P. S. Kozuch, G. N. Hortobagyi, and R. H. Blum, "Comparison of cardiac events associated with liposomal doxorubicin, epirubicin and doxorubicin in breast cancer: a Bayesian network meta-analysis," European Journal of Cancer, vol. 51, no. 16, pp. 2314-2320, 2015.

[3] T. V. Jerjian, A. E. Glode, L. A. Thompson, and C. L. O’Bryant, "Antibody-drug conjugates: a clinical pharmacy perspective on an emerging cancer therapy," Pharmacotherapy: The Journal of Human Pharmacology and Drug Therapy, vol. 36, no. 1, pp. 99116, 2016.

[4] A. R. Lehenbauer Ludke, A. A.-R. S. Al-Shudiefat, S. Dhingra, D. S. Jassal, and P. K. Singal, "A concise description of cardioprotective strategies in doxorubicin-induced cardiotoxicity," Canadian Journal of Physiology and Pharmacology, vol. 87, no. 10, pp. 756-763, 2009.

[5] B. L. Asselin, M. Devidas, L. Chen et al. et al., "Cardioprotection and safety of dexrazoxane in patients treated for newly diagnosed t-cell acute lymphoblastic leukemia or advancedstage lymphoblastic non-hodgkin lymphoma: a report of the children's oncology group randomized trial pediatric oncology group 9404," Journal of Clinical Oncology, vol. 34, no. 8, pp. 854$862,2016$.

[6] A. Tashakori Beheshti, H. Mostafavi Toroghi, G. Hosseini, A. Zarifian, F. Homaei Shandiz, and A. Fazlinezhad, "Carvedilol administration can prevent doxorubicin-induced cardiotoxicity: a double-blind randomized trial," Cardiology, vol. 134, no. 1, pp. 47-53, 2016.

[7] S. E. Lipshultz, V. I. Franco, T. L. Miller, S. D. Colan, and S. E. Sallan, "Cardiovascular disease in adult survivors of childhood cancer," Annual Review of Medicine, vol. 66, pp. 161-176, 2015.

[8] C.-J. Weng and G.-C. Yen, "Chemopreventive effects of dietary phytochemicals against cancer invasion and metastasis: phenolic acids, monophenol, polyphenol, and their derivatives," Cancer Treatment Reviews, vol. 38, no. 1, pp. 76-87, 2012.

[9] G. Wen, X.-X. Qu, D. Wang et al., "Recent advances in design, synthesis and bioactivity of paclitaxel-mimics," Fitoterapia, vol. 110, pp. 26-37, 2016.

[10] W. Wang and Y.-S. Liang, "Artemisinin: a wonder drug from Chinese natural medicines," Chinese Journal of Natural Medicines, vol. 14, no. 1, pp. 5-6, 2016.

[11] P. Angsutararux, S. Luanpitpong, and S. Issaragrisil, "Chemotherapy-induced cardiotoxicity: overview of the roles of oxidative stress," Oxidative Medicine and Cellular Longevity, vol. 2015, Article ID 795602, 13 pages, 2015.

[12] Z. V. Varga, P. Ferdinandy, L. Liaudet, and P. Pacher, "Druginduced mitochondrial dysfunction and cardiotoxicity," American Journal of Physiology-Heart and Circulatory Physiology, vol. 309, no. 9, pp. H1453-H1467, 2015.
[13] J. Ghosh, J. Das, P. Manna, and P. C. Sil, "The protective role of arjunolic acid against doxorubicin induced intracellular ROS dependent JNK-p38 and p53-mediated cardiac apoptosis," Biomaterials, vol. 32, no. 21, pp. 4857-4866, 2011.

[14] E. H. Choi, H.-J. Chang, J. Y. Cho, and H. S. Chun, "Cytoprotective effect of anthocyanins against doxorubicin-induced toxicity in $\mathrm{H} 9 \mathrm{c} 2$ cardiomyocytes in relation to their antioxidant activities," Food and Chemical Toxicology, vol. 45, no. 10, pp. 1873-1881, 2007.

[15] M. Saeed, O. Kadioglu, H. Khalid, Y. Sugimoto, and T. Efferth, "Activity of the dietary flavonoid, apigenin, against multidrugresistant tumor cells as determined by pharmacogenomics and molecular docking," Journal of Nutritional Biochemistry, vol. 26, no. 1, pp. 44-56, 2015.

[16] J. Psotová, Š. Chlopčíkova, P. Miketová, J. Hrbác, and V. Šimánek, "Chemoprotective effect of plant phenolics against anthracycline-induced toxicity on rat cardiomyocytes. Part III. Apigenin, baicalelin, kaempherol, luteolin and quercetin," Phytotherapy Research, vol. 18, no. 7, pp. 516-521, 2004.

[17] S.-Q. Wang, X.-F. Zhu, X.-N. Wang, T. Shen, F. Xiang, and H.$\mathrm{X}$. Lou, "Flavonoids from Malus hupehensis and their cardioprotective effects against doxorubicin-induced toxicity in H9c2 cells," Phytochemistry, vol. 87, pp. 119-125, 2013.

[18] Z. Li, Y.-N. Geng, J.-D. Jiang, and W.-J. Kong, "Antioxidant and anti-inflammatory activities of berberine in the treatment of diabetes mellitus," Evidence-Based Complementary and Alternative Medicine, vol. 2014, Article ID 289264, 12 pages, 2014.

[19] X. Lv, X. Yu, Y. Wang et al., "Berberine inhibits doxorubicintriggered cardiomyocyte apoptosis via attenuating mitochondrial dysfunction and increasing Bcl-2 expression," PLOS ONE, vol. 7, no. 10, Article ID e47351, 2012.

[20] G. Hao, Y. Yu, B. Gu, Y. Xing, and M. Xue, "Protective effects of berberine against doxorubicin-induced cardiotoxicity in rats by inhibiting metabolism of doxorubicin," Xenobiotica, vol. 45, no. 11, pp. 1024-1029, 2015.

[21] E. Ciesielska, A. Gwardys, and D. Metodiewa, "Anticancer, antiradical and antioxidative actions of novel Antoksyd S and its major components, baicalin and baicalein," Anticancer Research, vol. 22, no. 5, pp. 2885-2892, 2002.

[22] W.-T. Chang, J. Li, H.-H. Haung et al., "Baicalein protects against doxorubicin-induced cardiotoxicity by attenuation of mitochondrial oxidant injury and JNK activation," Journal of Cellular Biochemistry, vol. 112, no. 10, pp. 2873-2881, 2011.

[23] B. D. Sahu, J. M. Kumar, M. Kuncha, R. M. Borkar, R. Srinivas, and R. Sistla, "Baicalein alleviates doxorubicin-induced cardiotoxicity via suppression of myocardial oxidative stress and apoptosis in mice," Life Sciences, vol. 144, pp. 8-18, 2016.

[24] P. Zhang, Y. Tang, N.-G. Li, Y. Zhu, and J.-A. Duan, "Bioactivity and chemical synthesis of caffeic acid phenethyl ester and its derivatives," Molecules, vol. 19, no. 10, pp. 16458-16476, 2014.

[25] E. Fadillioglu, E. Oztas, H. Erdogan et al., "Protective effects of caffeic acid phenethyl ester on doxorubicin-induced cardiotoxicity in rats," Journal of Applied Toxicology, vol. 24, no. 1, pp. 47$52,2004$.

[26] J. G. Sena Filho, S. L. Nimmo, H. S. Xavier, J. M. Barbosa-Filho, and R. H. Cichewicz, "Phenylethanoid and lignan glycosides from polar extracts of Lantana, a genus of verbenaceous plants widely used in traditional herbal therapies," Journal of Natural Products, vol. 72, no. 7, pp. 1344-1347, 2009.

[27] D.-S. Kim, H.-R. Kim, E.-R. Woo et al., "Protective effect of calceolarioside on adriamycin-induced cardiomyocyte toxicity," 
European Journal of Pharmacology, vol. 541, no. 1-2, pp. 24-32, 2006.

[28] R. Durst, H. Danenberg, R. Gallily et al., "Cannabidiol, a nonpsychoactive Cannabis constituent, protects against myocardial ischemic reperfusion injury," American Journal of Physiology-Heart and Circulatory Physiology, vol. 293, no. 6, pp. H3602-H3607, 2007.

[29] A. A. Fouad, W. H. Albuali, A. S. Al-Mulhim, and I. Jresat, "Cardioprotective effect of cannabidiol in rats exposed to doxorubicin toxicity," Environmental Toxicology and Pharmacology, vol. 36, no. 2, pp. 347-357, 2013.

[30] E. Hao, P. Mukhopadhyay, Z. Cao et al., "Cannabidiol protects against doxorubicin-induced cardiomyopathy by modulating mitochondrial function and biogenesis," Molecular Medicine, vol. 21, pp. 38-45, 2015.

[31] M. Rajesh, P. Mukhopadhyay, S. Btkai et al., "Cannabidiol attenuates cardiac dysfunction, oxidative stress, fibrosis, and inflammatory and cell death signaling pathways in diabetic cardiomyopathy," Journal of the American College of Cardiology, vol. 56, no. 25, pp. 2115-2125, 2010.

[32] B. Horvth, P. Mukhopadhyay, G. Hask, and P. Pacher, "The endocannabinoid system and plant-derived cannabinoids in diabetes and diabetic complications," American Journal of Pathology, vol. 180, no. 2, pp. 432-442, 2012.

[33] M. Pennant, M. Steur, C. Moore, A. Butterworth, and L. Johnson, "Comparative validity of vitamin $\mathrm{C}$ and carotenoids as indicators of fruit and vegetable intake: a systematic review and meta-analysis of randomised controlled trials," British Journal of Nutrition, vol. 114, no. 9, pp. 1331-1340, 2015.

[34] R. Indu, T. S. Azhar, A. Nair, and C. K. K. Nair, "Amelioration of doxorubicin induced cardio-and hepato-toxicity by carotenoids," Journal of Cancer Research and Therapeutics, vol. 10, no. 1, pp. 62-67, 2014.

[35] S. F. Nabavi, N. Braidy, S. Habtemariam et al., "Neuroprotective effects of chrysin: from chemistry to medicine," Neurochemistry International, vol. 90, pp. 224-231, 2015.

[36] E. M. Mantawy, W. M. El-Bakly, A. Esmat, A. M. Badr, and E. ElDemerdash, "Chrysin alleviates acute doxorubicin cardiotoxicity in rats via suppression of oxidative stress, inflammation and apoptosis," European Journal of Pharmacology, vol. 728, no. 1, pp. 107-118, 2014.

[37] H.-S. Kim, M. J. Quon, and J.-A. Kim, "New insights into the mechanisms of polyphenols beyond antioxidant properties; lessons from the green tea polyphenol, epigallocatechin 3gallate," Redox Biology, vol. 2, pp. 187-195, 2014.

[38] S. Hrelia, A. Bordoni, C. Angeloni et al., "Green tea extracts can counteract the modification of fatty acid composition induced by doxorubicin in cultured cardiomyocytes," Prostaglandins Leukotrienes and Essential Fatty Acids, vol. 66, no. 5-6, pp. 519$524,2002$.

[39] W. Li, S. Nie, M. Xie, Y. Chen, C. Li, and H. Zhang, "A major green tea component, (-)-epigallocatechin-3-gallate, ameliorates doxorubicin-mediated cardiotoxicity in cardiomyocytes of neonatal rats," Journal of Agricultural and Food Chemistry, vol. 58, no. 16, pp. 8977-8982, 2010.

[40] J. Zheng, H. C. M. Lee, M. M. bin Sattar, Y. Huang, and J.S. Bian, "Cardioprotective effects of epigallocatechin-3-gallate against doxorubicin-induced cardiomyocyte injury," European Journal of Pharmacology, vol. 652, no. 1-3, pp. 82-88, 2011.

[41] N. M. Saeed, R. N. El-Naga, W. M. El-Bakly, H. M. Abdel-Rahman, R. A. Salah Eldin, and E. El-Demerdash,
"Epigallocatechin-3-gallate pretreatment attenuates doxorubicin-induced cardiotoxicity in rats: a mechanistic study," Biochemical Pharmacology, vol. 95, no. 3, pp. 145-155, 2015.

[42] J. Dudka, J. Jodynis-Liebert, E. Korobowicz et al., "Activity of NADPH-cytochrome P-450 reductase of the human heart, liver and lungs in the presence of (-)-epigallocatechin gallate, quercetin and resveratrol: an in vitro study," Basic \& Clinical Pharmacology \& Toxicology, vol. 97, no. 2, pp. 74-79, 2005.

[43] Z. Liu, X.-D. Song, Y. Xin et al., "Protective effect of chrysoeriol against doxorubicin-induced cardiotoxicity in vitro," Chinese Medical Journal, vol. 122, no. 21, pp. 2652-2656, 2009.

[44] S. Prasad, A. K. Tyagi, and B. B. Aggarwal, "Recent developments in delivery, bioavailability, absorption and metabolism of curcumin: the golden pigment from golden spice," Cancer Research and Treatment, vol. 46, no. 1, pp. 2-18, 2014.

[45] N. Venkatesan, "Curcumin attenuation of acute adriamycin myocardial toxicity in rats," British Journal of Pharmacology, vol. 124, no. 3, pp. 425-427, 1998.

[46] A. Dayton, K. Selvendiran, S. Meduru et al., "Amelioration of doxorubicin-induced cardiotoxicity by an anticancerantioxidant dual-function compound, HO-3867," Journal of Pharmacology and Experimental Therapeutics, vol. 339, no. 2, pp. 350-357, 2011.

[47] L. Hosseinzadeh, J. Behravan, F. Mosaffa, G. Bahrami, A. Bahrami, and G. Karimi, "Curcumin potentiates doxorubicininduced apoptosis in $\mathrm{H} 9 \mathrm{c} 2$ cardiac muscle cells through generation of reactive oxygen species," Food and Chemical Toxicology, vol. 49, no. 5, pp. 1102-1109, 2011.

[48] A. V. Swamy, S. Gulliaya, A. Thippeswamy, B. C. Koti, and D. V. Manjula, "Cardioprotective effect of curcumin against doxorubicin-induced myocardial toxicity in albino rats," Indian Journal of Pharmacology, vol. 44, no. 1, pp. 73-77, 2012.

[49] D. Pramanik, N. R. Campbell, S. Das et al., "A composite polymer nanoparticle overcomes multidrug resistance and ameliorates doxorubicin-associated cardiomyopathy," Oncotarget, vol. 3, no. 6, pp. 640-650, 2012.

[50] K. Pramod, S. H. Ansari, and J. Ali, "Eugenol: a natural compound with versatile pharmacological actions," Natural Product Communications, vol. 5, no. 12, pp. 1999-2006, 2010.

[51] A. A. Fouad and M. T. Yacoubi, "Mechanisms underlying the protective effect of eugenol in rats with acute doxorubicin cardiotoxicity," Archives of Pharmacal Research, vol. 34, no. 5, pp. 821-828, 2011.

[52] F. A. A. van Acker, E. Boven, K. Kramer, G. R. M. M. Haenen, A. Bast, and W. J. F. van der Vijgh, "Frederine, a new and promising protector against doxorubicin-induced cardiotoxicity," Clinical Cancer Research, vol. 7, no. 5, pp. 1378-1384, 2001.

[53] F. A. A. van Acker, S. A. B. E. van Acker, K. Kramer, G. R. M. M. Haenen, A. Bast, and W. J. F. van der Vijgh, "7-Monohydroxyethylrutoside protects against chronic doxorubicin-induced cardiotoxicity when administered only once per week," Clinical Cancer Research, vol. 6, no. 4, pp. 1337-1341, 2000.

[54] S. A. B. E. van Acker, E. Boven, K. Kuiper et al., "Monohydroxyethylrutoside, a dose-dependent cardioprotective agent, does not affect the antitumor activity of doxorubicin," Clinical Cancer Research, vol. 3, no. 10, pp. 1747-1754, 1997.

[55] A. A. Oyagbemi, A. B. Saba, and O. I. Azeez, "Molecular targets of [6]-gingerol: its potential roles in cancer chemoprevention," Biofactors, vol. 36, no. 3, pp. 169-178, 2010. 
[56] W. M. El-Bakly, M. L. Louka, A. M. El-Halawany, and M. F. Schaalan, "6-Gingerol ameliorated doxorubicin-induced cardiotoxicity: role of nuclear factor kappa B and protein glycation," Cancer Chemotherapy and Pharmacology, vol. 70, no. 6, pp. 833-841, 2012.

[57] S. F. Nabavi, A. Sureda, S. Habtemariam, and S. M. Nabavi, "Ginsenoside Rd and ischemic stroke; a short review of literatures," Journal of Ginseng Research, vol. 39, no. 4, pp. 299-303, 2015.

[58] H. Wang, P. Yu, H. Gou et al., "Cardioprotective effects of 20(S)ginsenoside $\mathrm{Rh} 2$ against doxorubicin-induced cardiotoxicity in vitro and in vivo," Evidence-Based Complementary and Alternative Medicine, vol. 2012, Article ID 506214, 8 pages, 2012.

[59] C.-T. Chen, Z.-H. Wang, C.-C. Hsu, H.-H. Lin, and J.-H. Chen, "In vivo protective effects of diosgenin against doxorubicininduced cardiotoxicity," Nutrients, vol. 7, no. 6, pp. 4938-4954, 2015.

[60] F. Zhou, G. Hao, J. Zhang et al., "Protective effect of 23hydroxybetulinic acid on doxorubicin-induced cardiotoxicity: a correlation with the inhibition of carbonyl reductase-mediated metabolism," British Journal of Pharmacology, vol. 172, no. 23, pp. 5690-5703, 2015.

[61] A. Roohbakhsh, H. Parhiz, F. Soltani, R. Rezaee, and M. Iranshahi, "Molecular mechanisms behind the biological effects of hesperidin and hesperetin for the prevention of cancer and cardiovascular diseases," Life Sciences, vol. 124, pp. 64-74, 2015.

[62] I. T. Abdel-Raheem and A. A. Abdel-Ghany, "Hesperidin alleviates doxorubicin-induced cardiotoxicity in rats," Journal of the Egyptian National Cancer Institute, vol. 21, no. 2, pp. 175-184, 2009.

[63] N. F. Khedr and R. M. Khalil, "Effect of hesperidin on mice bearing Ehrlich solid carcinoma maintained on doxorubicin," Tumor Biology, vol. 36, no. 12, pp. 9267-9275, 2015.

[64] P. P. Trivedi, S. Kushwaha, D. N. Tripathi, and G. B. Jena, "Cardioprotective effects of hesperetin against doxorubicininduced oxidative stress and DNA damage in rat," Cardiovascular Toxicology, vol. 11, no. 3, pp. 215-225, 2011.

[65] S. Granados-Principal, N. El-Azem, R. Pamplona et al., "Hydroxytyrosol ameliorates oxidative stress and mitochondrial dysfunction in doxorubicin-induced cardiotoxicity in rats with breast cancer," Biochemical Pharmacology, vol. 90, no. 1, pp. 25-33, 2014.

[66] J. Sun, G. Sun, X. Meng et al., "Isorhamnetin protects against doxorubicin-induced cardiotoxicity in vivo and in vitro," PLoS ONE, vol. 8, no. 5, Article ID e64526, 2013.

[67] A. A. Adwas, A. A. Elkhoely, A. M. Kabel, M. N. AbdelRahman, and A. A. Eissa, "Anti-cancer and cardioprotective effects of indol-3-carbinol in doxorubicin-treated mice," Journal of Infection and Chemotherapy, vol. 22, no. 1, pp. 36-43, 2016.

[68] P. Souček, E. Kondrová, J. Heřmánek et al., "New model system for testing effects of flavonoids on doxorubicin-related formation of hydroxyl radicals," Anti-Cancer Drugs, vol. 22, no. 2, pp. 176-184, 2011.

[69] J. Xiao, G.-B. Sun, B. Sun et al., "Kaempferol protects against doxorubicin-induced cardiotoxicity in vivo and in vitro," Toxicology, vol. 292, no. 1, pp. 53-62, 2012.

[70] G. Karimi, M. Ramezani, and A. Abdi, "Protective effects of lycopene and tomato extract against doxorubicin-induced cardiotoxicity," Phytotherapy Research, vol. 19, no. 10, pp. 912914, 2005.

[71] S. Yilmaz, A. Atessahin, E. Sahna, I. Karahan, and S. Ozer, "Protective effect of lycopene on adriamycin-induced cardiotoxicity and nephrotoxicity," Toxicology, vol. 218, no. 2-3, pp. 164-171, 2006.

[72] A. L. A. Ferreira, D. M. F. Salvadori, M. C. M. O. Nascimento et al., "Tomato-oleoresin supplement prevents doxorubicininduced cardiac myocyte oxidative DNA damage in rats," Mutation Research/Genetic Toxicology and Environmental Mutagenesis, vol. 631, no. 1, pp. 26-35, 2007.

[73] A. L. A. Ferreira, K.-J. Yeum, L. S. Matsubara et al., "Doxorubicin as an antioxidant: maintenance of myocardial levels of lycopene under doxorubicin treatment," Free Radical Biology and Medicine, vol. 43, no. 5, pp. 740-751, 2007.

[74] K. Sahin, N. Sahin, and O. Kucuk, "Lycopene and chemotherapy toxicity," Nutrition and Cancer, vol. 62, no. 7, pp. 988-995, 2010.

[75] S.-Q. Wang, X.-Z. Han, X. Li, D.-M. Ren, X.-N. Wang, and H.$\mathrm{X}$. Lou, "Flavonoids from Dracocephalum tanguticum and their cardioprotective effects against doxorubicin-induced toxicity in H9c2 cells," Bioorganic \& Medicinal Chemistry Letters, vol. 20, no. 22, pp. 6411-6415, 2010.

[76] H. Yao, Z. Shang, P. Wang et al., "Protection of luteolin7-O-glucoside against doxorubicin-induced injury through PTEN/Akt and ERK pathway in H9c2 cells," Cardiovascular Toxicology, vol. 16, no. 2, pp. 101-110, 2016.

[77] L. D. S. Kok, Y. P. Wong, T. W. Wu, H. C. Chan, T. T. Kwok, and K. P. Fung, "Morin hydrate: a potential antioxidant in minimizing the free-radicals- mediated damage to cardiovascular cells by anti-tumor drugs," Life Sciences, vol. 67, no. 1, pp. 91-99, 2000.

[78] W. Arozal, F. D. Suyatna, V. Juniantito et al., "The effects of mangiferin (Mangifera indica L) in doxorubicin-induced cardiotoxicity in rats," Drug Research, vol. 65, no. 11, pp. 574580, 2015.

[79] F. D. Agustini, W. Arozal, M. Louisa et al., "Cardioprotection mechanism of mangiferin on doxorubicin-induced rats: focus on intracellular calcium regulation," Pharmaceutical Biology, In press.

[80] H.-S. Park, J. U.-H. Oh, J. H. Lee, and Y.-J. Lee, "Minor effects of the citrus flavonoids naringin, naringenin and quercetin, on the pharmacokinetics of doxorubicin in rats," Pharmazie, vol. 66, no. 6, pp. 424-429, 2011.

[81] H. M. Arafa, M. F. Abd-Ellah, and H. F. Hafez, "Abatement by naringenin of doxorubicin-induced cardiac toxicity in rats," Journal of the Egyptian National Cancer Institute, vol. 17, no. 4, pp. 291-300, 2005.

[82] S. S. Shiromwar and V. R. Chidrawar, "Combined effects of p-coumaric acid and naringenin against doxorubicin-induced cardiotoxicity in rats," Pharmacognosy Research, vol. 3, no. 3, pp. 214-219, 2011.

[83] X. Han, J. Pan, D. Ren, Y. Cheng, P. Fan, and H. Lou, "Naringenin-7-O-glucoside protects against doxorubicininduced toxicity in $\mathrm{H} 9 \mathrm{c} 2$ cardiomyocytes by induction of endogenous antioxidant enzymes," Food and Chemical Toxicology, vol. 46, no. 9, pp. 3140-3146, 2008.

[84] X. Han, D. Ren, P. Fan, T. Shen, and H. Lou, "Protective effects of naringenin-7-O-glucoside on doxorubicin-induced apoptosis in H9C2 cells," European Journal of Pharmacology, vol. 581, no. 1-2, pp. 47-53, 2008.

[85] X. Han, S. Gao, Y. Cheng et al., "Protective effect of naringenin7-O-glucoside against oxidative stress induced by doxorubicin in H9c2 cardiomyocytes," BioScience Trends, vol. 6, no. 1, pp. 1925, 2012.

[86] X. Fu, L. Kong, M. Tang et al., "Protective effect of ocotillol against doxorubicin-induced acute and chronic cardiac injury," Molecular Medicine Reports, vol. 9, no. 1, pp. 360-364, 2014. 
[87] I. Andreadou, M. Papaefthimiou, A. Zira et al., "Metabonomic identification of novel biomarkers in doxorubicin cardiotoxicity and protective effect of the natural antioxidant oleuropein," NMR in Biomedicine, vol. 22, no. 6, pp. 585-592, 2009.

[88] I. Andreadou, F. Sigala, E. K. Iliodromitis et al., "Acute doxorubicin cardiotoxicity is successfully treated with the phytochemical oleuropein through suppression of oxidative and nitrosative stress," Journal of Molecular and Cellular Cardiology, vol. 42, no. 3, pp. 549-558, 2007.

[89] I. Andreadou, E. Mikros, K. Ioannidis et al., "Oleuropein prevents doxorubicin-induced cardiomyopathy interfering with signaling molecules and cardiomyocyte metabolism," Journal of Molecular and Cellular Cardiology, vol. 69, pp. 4-16, 2014.

[90] H. Xu, Y. Han, M. Zhang, M. Yan, and C. Gao, "Protective role of Osthole on myocardial cell apoptosis induced by doxorubicin in rats," International Journal of Clinical and Experimental Pathology, vol. 8, no. 9, pp. 10816-10823, 2015.

[91] M. H. Abdel-Wahab, M. A. El-Mahdy, M. F. Abd-Ellah, G. K. Helal, F. Khalifa, and F. M. A. Hamada, "Influence of pcoumaric acid on doxorubicin-induced oxidative stress in rat's heart," Pharmacological Research, vol. 48, no. 5, pp. 461-465, 2003.

[92] S. Panda and A. Kar, "Periplogenin-3-O- -D-Glucopyranosyl $(1 \rightarrow 6)$ - -D-glucopyaranosyl- - $(1 \rightarrow 4)$-D-cymaropyranoside, isolated from Aegle marmelos protects doxorubicin induced cardiovascular problems and hepatotoxicity in rats," Cardiovascular Therapeutics, vol. 27, no. 2, pp. 108-116, 2009.

[93] D.-S. Kim, E.-R. Woo, S.-W. Chae et al., "Plantainoside D protects adriamycin-induced apoptosis in $\mathrm{H} 9 \mathrm{c} 2$ cardiac muscle cells via the inhibition of ROS generation and NF- $\kappa \mathrm{B}$ activation," Life Sciences, vol. 80, no. 4, pp. 314-323, 2007.

[94] M. Khan, S. Varadharaj, J. C. Shobha et al., "C-phycocyanin ameliorates doxorubicin-induced oxidative stress and apoptosis in adult rat cardiomyocytes," Journal of Cardiovascular Pharmacology, vol. 47, no. 1, pp. 9-20, 2006.

[95] A. Maimoona, I. Naeem, Z. Saddiqe, and K. Jameel, "A review on biological, nutraceutical and clinical aspects of French maritime pine bark extract," Journal of Ethnopharmacology, vol. 133, no. 2, pp. 261-277, 2011.

[96] N. A. E. Boghdady, "Antioxidant and antiapoptotic effects of proanthocyanidin and ginkgo biloba extract against doxorubicin-induced cardiac injury in rats," Cell Biochemistry and Function, vol. 31, no. 4, pp. 344-351, 2013.

[97] S. D. Ray, D. Patel, V. Wong, and D. Bagchi, "In vivo protection of DNA damage associated apoptotic and necrotic cell deaths during acetaminophen-induced nephrotoxicity, amiodaroneinduced lung toxicity and doxorubicin-induced cardiotoxicity by a novel IH636 grape seed proanthocyanidin extract," Research Communications in Molecular Pathology and Pharmacology, vol. 107, no. 1-2, pp. 137-166, 2000.

[98] D. Bagchi, S. D. Ray, M. Bagchi, H. G. Preuss, and S. J. Stohs, "Mechanistic pathways of antioxidant cytoprotection by a novel IH636 grape seed proanthocyanidin extract," Indian Journal of Experimental Biology, vol. 40, no. 6, pp. 717-726, 2002.

[99] X.-Y.Zhang, D.-C. Bai, Y.-J. Wu, W.-G. Li, and N.-F. Liu, "Proanthocyanidin from grape seeds enhances anti-tumor effect of doxorubicin both in vitro and in vivo," Pharmazie, vol. 60, no. 7, pp. 533-538, 2005.

[100] E. Demirkaya, A. Avci, V. Kesik et al., "Cardioprotective roles of aged garlic extract, grape seed proanthocyanidin, and hazelnut on doxorubicin-induced cardiotoxicity," Canadian Journal of Physiology and Pharmacology, vol. 87, no. 8, pp. 633-640, 2009.
[101] C. Cal, H. Garban, A. Jazirehi, C. Yeh, Y. Mizutani, and B. Bonavida, "Resveratrol and cancer: chemoprevention, apoptosis, and chemo-immunosensitizing activities," Current Medicinal Chemistry-Anti-Cancer Agents, vol. 3, no. 2, pp. 77-93, 2003.

[102] Z. Cao and Y. Li, "Potent induction of cellular antioxidants and phase 2 enzymes by resveratrol in cardiomyocytes: protection against oxidative and electrophilic injury," European Journal of Pharmacology, vol. 489, no. 1-2, pp. 39-48, 2004.

[103] E. Tatlidede, Ö. Şehirli, A. Velioğlu-Öğün et al., "Resveratrol treatment protects against doxorubicin-induced cardiotoxicity by alleviating oxidative damage," Free Radical Research, vol. 43, no. 3, pp. 195-205, 2009.

[104] G. Y. Wang, Y. M. Wang, L. N. Zhang et al., "Effect of resveratrol on heart function of rats with adriamycin-induced heart failure," Zhongguo Zhong Yao Za Zhi, vol. 32, no. 15, pp. 1563-1565, 2007.

[105] E. D. Brookins Danz, J. Skramsted, N. Henry, J. A. Bennett, and R. S. Keller, "Resveratrol prevents doxorubicin cardiotoxicity through mitochondrial stabilization and the Sirtl pathway," Free Radical Biology and Medicine, vol. 46, no. 12, pp. 1589-1597, 2009.

[106] C. Zhang, Y. Feng, S. Qu et al., "Resveratrol attenuates doxorubicin-induced cardiomyocyte apoptosis in mice through SIRT1-mediated deacetylation of p53," Cardiovascular Research, vol. 90, no. 3, pp. 538-545, 2011.

[107] X. Xu, K. Chen, S. Kobayashi, D. Timm, and Q. Liang, "Resveratrol attenuates doxorubicin-induced cardiomyocyte death via inhibition of p70 S6 kinase 1-mediated autophagy," Journal of Pharmacology and Experimental Therapeutics, vol. 341, no. 1, pp. 183-195, 2012.

[108] S. Wang, P. Song, and M.-H. Zou, "Inhibition of AMP-activated protein kinase $\alpha(\mathrm{AMPK} \alpha)$ by doxorubicin accentuates genotoxic stress and cell death in mouse embryonic fibroblasts and cardiomyocytes: role of $\mathrm{p} 53$ and SIRT1," The Journal of Biological Chemistry, vol. 287, no. 11, pp. 8001-8012, 2012.

[109] C.-Y. Chen, J.-H. Jang, M.-H. Li, and Y.-J. Surh, "Resveratrol upregulates heme oxygenase-1 expression via activation of NFE2-related factor 2 in PC12 cells," Biochemical and Biophysical Research Communications, vol. 331, no. 4, pp. 993-1000, 2005.

[110] J. Gu, Z.-P. Song, D.-M. Gui, W. Hu, Y.-G. Chen, and D.-D. Zhang, "Resveratrol attenuates doxorubicin-induced cardiomyocyte apoptosis in lymphoma nude mice by heme oxygenase1 induction," Cardiovascular Toxicology, vol. 12, no. 4, pp. 341349, 2012.

[111] F. A. Pinarli, N. N. Turan, F. G. Pinarli et al., "Resveratrol and adipose-derived mesenchymal stem cells are effective in the prevention and treatment of doxorubicin cardiotoxicity in rats," Pediatric Hematology and Oncology, vol. 30, no. 3, pp. 226-238, 2013.

[112] V. W. Dolinsky, K. J. Rogan, M. M. Sung et al., "Both aerobic exercise and resveratrol supplementation attenuate doxorubicin-induced cardiac injury in mice," American Journal of Physiology-Endocrinology and Metabolism, vol. 305, no. 2, pp. E243-E253, 2013.

[113] M. G. Novelle, D. Wahl, C. Diéguez, M. Bernier, and R. de Cabo, "Resveratrol supplementation: where are we now and where should we go?” Ageing Research Reviews, vol. 21, 15 pages, 2015.

[114] P. A. Janeesh and A. Abraham, "Robinin modulates doxorubicin-induced cardiac apoptosis by TGF- $\hat{I}^{2} 1$ signaling pathway in Sprague Dawley rats," Biomedicine \& Pharmacotherapy, vol. 68, no. 8, pp. 989-998, 2014. 
[115] D.-S. Kim, H.-R. Kim, E.-R. Woo, S.-T. Hong, H.-J. Chae, and S.W. Chae, "Inhibitory effects of rosmarinic acid on adriamycininduced apoptosis in $\mathrm{H} 9 \mathrm{c} 2$ cardiac muscle cells by inhibiting reactive oxygen species and the activations of c-Jun $\mathrm{N}$-terminal kinase and extracellular signal-regulated kinase," Biochemical Pharmacology, vol. 70, no. 7, pp. 1066-1078, 2005.

[116] Š. Chlopčíková, J. Psotová, P. Miketová, J. Soušek, V. Lichnovský, and V. Šimánek, "Chemoprotective effect of plant phenolics against anthracycline-induced toxicity on rat cardiomyocytes part II. Caffeic, chlorogenic and rosmarinic acids," Phytotherapy Research, vol. 18, no. 5, pp. 408-413, 2004.

[117] B. Jiang, L. Zhang, M. Li et al., "Salvianolic acids prevent acute doxorubicin cardiotoxicity in mice through suppression of oxidative stress," Food and Chemical Toxicology, vol. 46, no. 5, pp. 1510-1515, 2008.

[118] T.-J. Lin, G.-T. Liu, Y. Liu, and G.-Z. Xu, "Protection by salvianolic acid A against adriamycin toxicity on rat heart mitochondria," Free Radical Biology and Medicine, vol. 12, no. 5, pp. 347-351, 1992.

[119] L. Li, Q. Pan, W. Han, Z. Liu, L. Li, and X. Hu, "Schisandrin B prevents doxorubicin-induced cardiotoxicity via enhancing glutathione redox cycling," Clinical Cancer Research, vol. 13, no. 22, pp. 6753-6760, 2007.

[120] F. Che, Y. Liu, and C. Xu, "Prevention and treatment of doxorubicin-induced cardiotoxicity by dexrazoxane and schisandrin B in rabbits," International Journal of Toxicology, vol. 30, no. 6, pp. 681-689, 2011.

[121] R. A. Thandavarayan, V. V. Giridharan, S. Arumugam et al., "Schisandrin b prevents doxorubicin induced cardiac dysfunction by modulation of DNA damage, oxidative stress and inflammation through inhibition of mapk/p53 signaling," PLoS ONE, vol. 10, no. 3, Article ID e0119214, 2015.

[122] Y. Xu, Z. Liu, J. Sun et al., "Schisandrin B prevents doxorubicininduced chronic cardiotoxicity and enhances its anticancer activity in vivo," PLoS ONE, vol. 6, no. 12, Article ID e28335, 2011.

[123] X.-L. Wang, X. Wang, L.-L. Xiong et al., "Salidroside improves doxorubicin-induced cardiac dysfunction by suppression of excessive oxidative stress and cardiomyocyte apoptosis," Journal of Cardiovascular Pharmacology, vol. 62, no. 6, pp. 512-523, 2013.

[124] S. Su, Q. Li, Y. liu et al., "Sesamin ameliorates doxorubicininduced cardiotoxicity: involvement of Sirtl and Mn-SOD pathway," Toxicology Letters, vol. 224, no. 2, pp. 257-263, 2014.

[125] A. Chennuru and M. T. S. Saleem, "Antioxidant, lipid lowering, and membrane stabilization effect of sesamol against doxorubicin-induced cardiomyopathy in experimental rats," BioMed Research International, vol. 2013, Article ID 934239, 5 pages, 2013.

[126] A. Raskovic, N. Stilinovic, J. Kolarovic, V. Vasovic, S. Vukmirovic, and M. Mikov, "The protective effects of silymarin against doxorubicin-induced cardiotoxicity and hepatotoxicity in rats," Molecules, vol. 16, no. 10, pp. 8601-8613, 2011.

[127] C. A. Houghton, R. G. Fassett, and J. S. Coombes, "Sulforaphane: translational research from laboratory bench to clinic," Nutrition Reviews, vol. 71, no. 11, pp. 709-726, 2013.

[128] B. Li, D. S. Kim, R. K. Yadav, H. R. Kim, and H. J. Chae, "Sulforaphane prevents doxorubicin-induced oxidative stress and cell death in rat $\mathrm{H} 9 \mathrm{c} 2$ cells," International Journal of Molecular Medicine, vol. 36, no. 1, pp. 53-64, 2015.

[129] P. Singh, R. Sharma, K. McElhanon, C. D. Allen, J. K. Megyesi, and H. Bene, "Sulforaphane protects the heart from doxorubicin-induced toxicity," Free Radical Biology and Medicine, vol. 86, pp. 90-101, 2015.

[130] H.-L. Tian, T. Yu, N.-N. Xu et al., "A novel compound modified from tanshinone inhibits tumor growth in vivo via activation of the intrinsic apoptotic pathway," Cancer Letters, vol. 297, no. 1, pp. 18-30, 2010.

[131] G.-Y. Zhou, B.-L. Zhao, J.-W. Hou, G.-E. Ma, and W.-J. Xin, "Protective effects of sodium tanshinone IIA sulphonate against adriamycin-induced lipid peroxidation in mice hearts in vivo and in vitro," Pharmacological Research, vol. 40, no. 6, pp. 487491, 1999.

[132] J. Gao, G. Yang, R. Pi et al., "Tanshinone IIA protects neonatal rat cardiomyocytes from adriamycin-induced apoptosis," Translational Research, vol. 151, no. 2, pp. 79-87, 2008.

[133] B. Jiang, L. Zhang, Y. Wang et al., "Tanshinone IIA sodium sulfonate protects against cardiotoxicity induced by doxorubicin in vitro and in vivo," Food and Chemical Toxicology, vol. 47, no. 7, pp. 1538-1544, 2009.

[134] H.-J. Hong, J.-C. Liu, P.-Y. Chen, J.-J. Chen, P. Chan, and T.-H. Cheng, "Tanshinone IIA prevents doxorubicin-induced cardiomyocyte apoptosis through Akt-dependent pathway," International Journal of Cardiology, vol. 157, no. 2, pp. 174-179, 2012.

[135] S.-H. Zhang, W.-Q. Wang, and J.-L. Wang, "Protective effect of tetrahydroxystilbene glucoside on cardiotoxicity induced by doxorubicin in vitro and in vivo," Acta Pharmacologica Sinica, vol. 30, no. 11, pp. 1479-1487, 2009.

[136] C. C. Woo, S. Y. Loo, V. Gee et al., "Anticancer activity of thymoquinone in breast cancer cells: possible involvement of PPAR- $\gamma$ pathway," Biochemical Pharmacology, vol. 82, no. 5, pp. 464-475, 2011.

[137] O. A. Al-Shabanah, O. A. Badary, M. N. Nagi, N. M. AlGharably, A. C. Al-Rikabi, and A. M. Al-Bekairi, "Thymoquinone protects against doxorubicin-induced cardiotoxicity without compromising its antitumor activity," Journal of Experimental and Clinical Cancer Research, vol. 17, no. 2, pp. 193-198, 1998.

[138] M. N. Nagi and M. A. Mansour, "Protective effect of thymoquinone against doxorubicin-induced cardiotoxicity in rats: a possible mechanism of protection," Pharmacological Research, vol. 41, no. 3, pp. 283-289, 2000.

[139] R. K. Brown, G. Wilson, M. A. Tucci, and H. A. Benghuzzi, "The effects of thymoquinone and Doxorubicin on leukemia and cardiomyocyte cell lines," Biomedical Sciences Instrumentation, vol. 50, pp. 391-396, 2014.

[140] M. Xu, L. Sheng, X. Zhu, S. Zeng, D. Chi, and G.-J. Zhang, "Protective effect of tetrandrine on doxorubicin-induced cardiotoxicity in rats," Tumori, vol. 96, no. 3, pp. 460-464, 2010.

[141] C.-L. Dai, H.-Y. Xiong, L.-F. Tang et al., "Tetrandrine achieved plasma concentrations capable of reversing MDR in vitro and had no apparent effect on doxorubicin pharmacokinetics in mice," Cancer Chemotherapy and Pharmacology, vol. 60, no. 5, pp. 741-750, 2007.

[142] R. Deng, "Therapeutic effects of guggul and its constituent guggulsterone: cardiovascular benefits," Cardiovascular Drug Reviews, vol. 25, no. 4, pp. 375-390, 2007.

[143] W.-C. Wang, Y.-H. Uen, M.-L. Chang et al., "Protective effect of guggulsterone against cardiomyocyte injury induced by doxorubicin in vitro," BMC Complementary and Alternative Medicine, vol. 12, no. 1, article 138, 10 pages, 2012.

[144] K. Chatterjee, J. Zhang, R. Tao, N. Honbo, and J. S. Karliner, "Vincristine attenuates doxorubicin cardiotoxicity," Biochemical 
and Biophysical Research Communications, vol. 373, no. 4, pp. 555-560, 2008.

[145] L. Xi, "Visnagin-a new protectant against doxorubicin cardiotoxicity? Inhibition of mitochondrial malate dehydrogenase 2 (MDH2) and beyond," Annals of Translational Medicine, vol. 4, no. 4, p. 65, 2016.

[146] Y. Liu, A. Asnani, L. Zou et al., "Visnagin protects against doxorubicin-induced cardiomyopathy through modulation of mitochondrial malate dehydrogenase," Science Translational Medicine, vol. 6, no. 266, Article ID 266ral70, 2014.

[147] P. Anand, A. B. Kunnumakkara, R. A. Newman, and B. B. Aggarwal, "Bioavailability of curcumin: problems and promises," Molecular Pharmaceutics, vol. 4, no. 6, pp. 807-818, 2007.

[148] M. Airoldi, D. Amadori, S. Barni et al., "Clinical activity and cardiac tolerability of non-pegylated liposomal doxorubicin in breast cancer: a synthetic review," Tumori, vol. 97, no. 6, pp. 690692, 2011. 


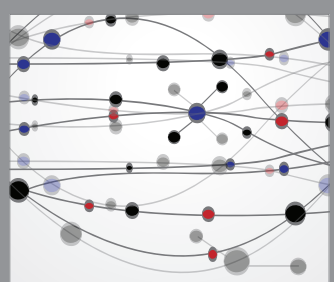

The Scientific World Journal
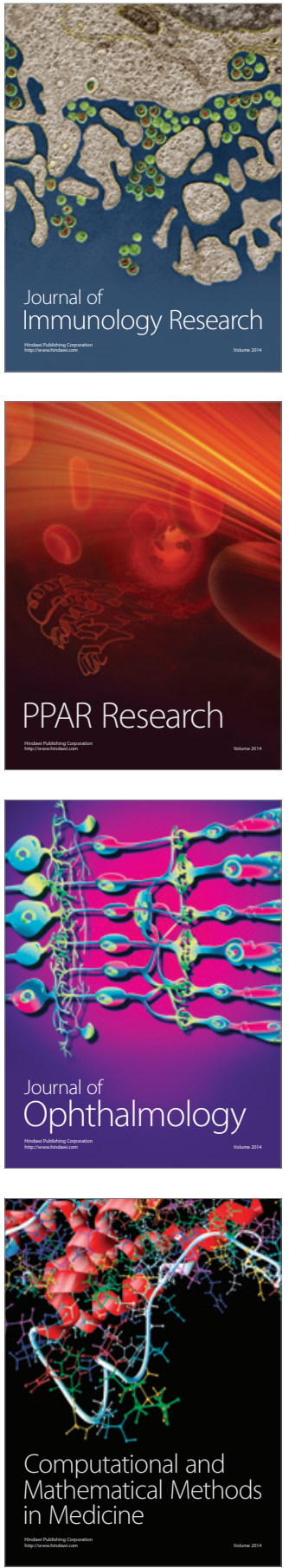

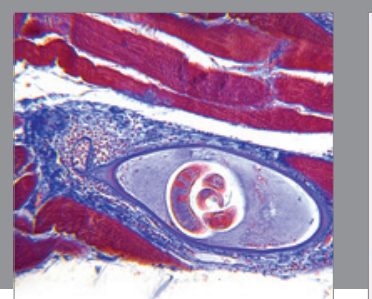

Gastroenterology Research and Practice

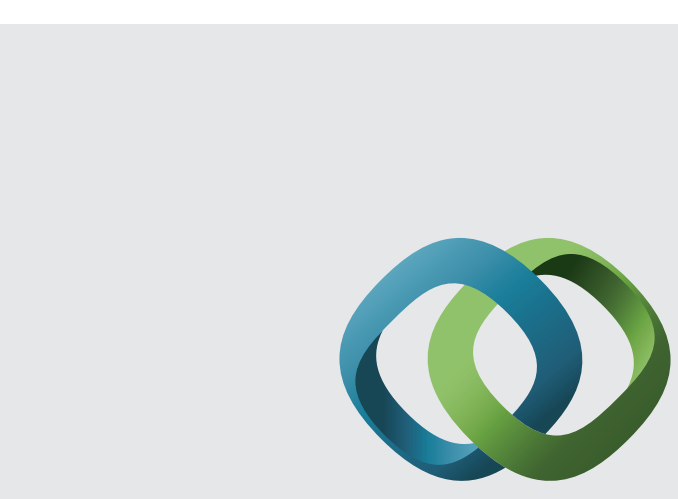

\section{Hindawi}

Submit your manuscripts at

http://www.hindawi.com
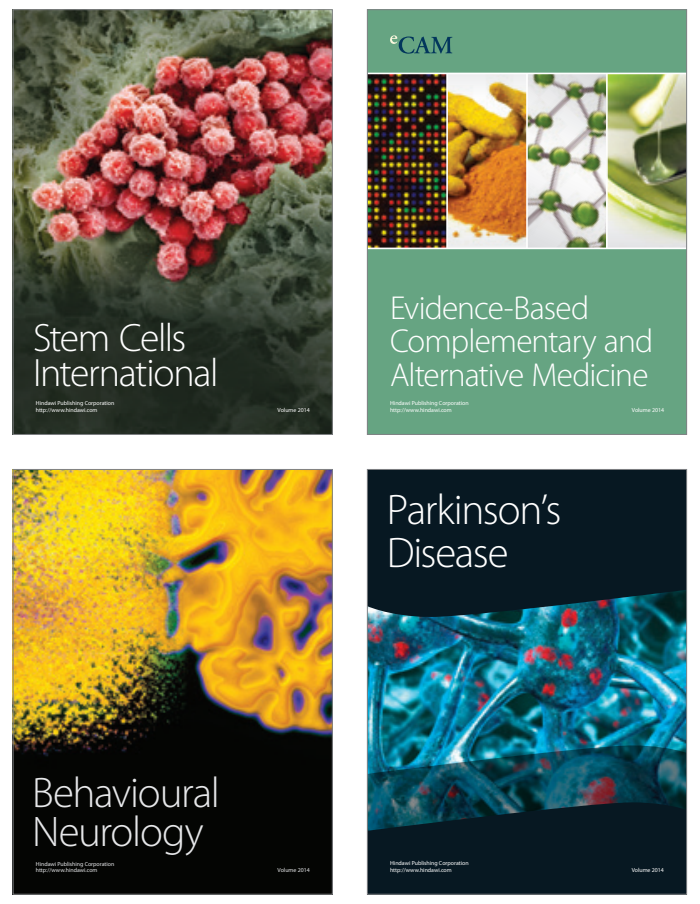
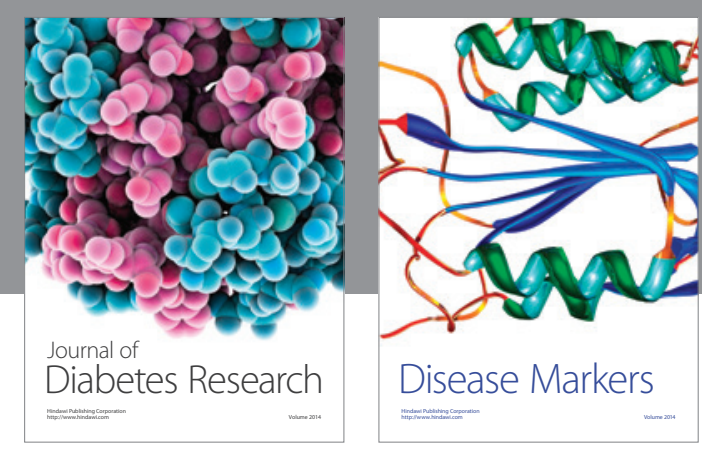

Disease Markers
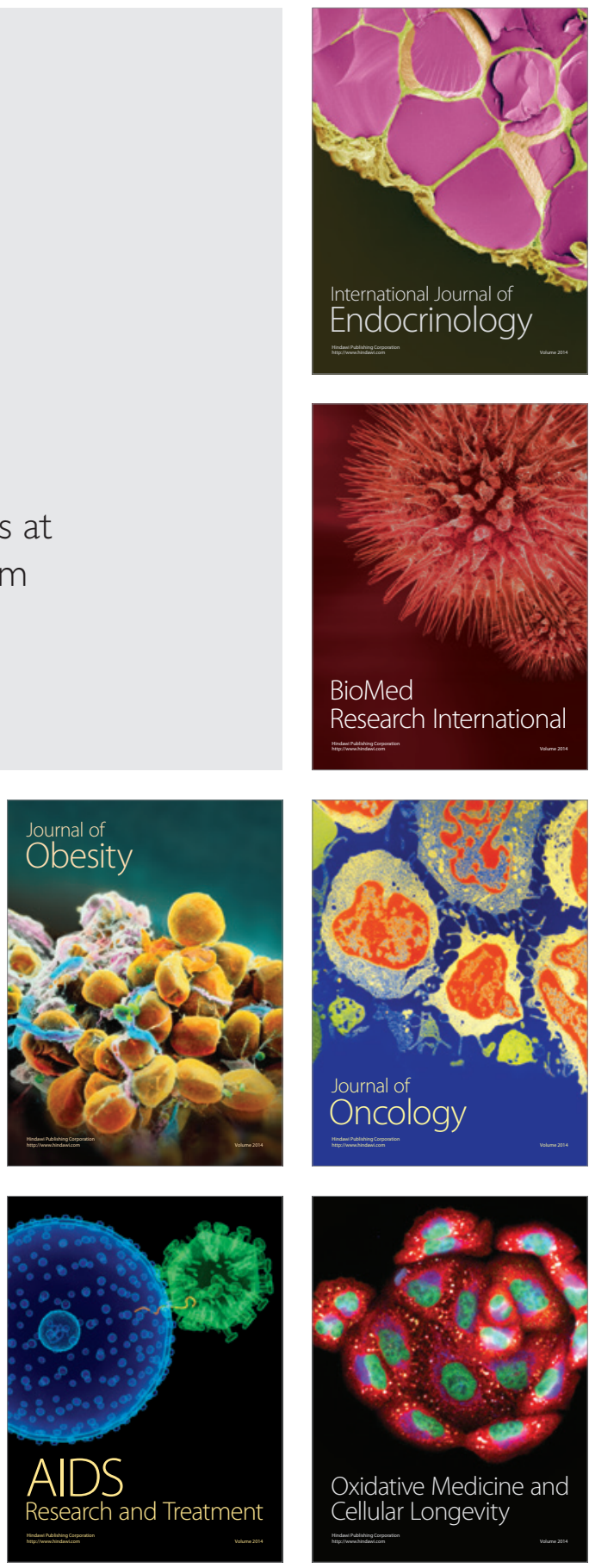